\title{
RPA and Pif1 cooperate to remove G-rich structures at both leading and lagging strand
}

\author{
Laetitia Maestroni ${ }^{1}$, Julien Audry ${ }^{1}$, Pierre Luciano ${ }^{1}$, Stéphane Coulon ${ }^{1}$, Vincent Géli ${ }^{1, *}$ and Yves Corda $^{1, *}$ \\ ${ }^{1}$ Aix-Marseille Univ, Inserm, CNRS, Institut Paoli-Calmettes, CRCM, Marseille, France. Equipe Labellisée par la Ligue Nationale contre \\ le Cancer. \\ * Corresponding Authors: \\ Vincent Géli, Aix-Marseille Univ, Inserm, CNRS, Institut Paoli-Calmettes, CRCM, Marseille, France. Equipe Labellisée par la Ligue \\ Nationale contre le Cancer ; E-mail: vincent.geli@inserm.fr; \\ Yves Corda, Aix-Marseille Univ, Inserm, CNRS, Institut Paoli-Calmettes, CRCM, Marseille, France. Equipe Labellisée par la Ligue \\ Nationale contre le Cancer ; E-mail: yves.corda@inserm.fr
}

\begin{abstract}
In Saccharomyces cerevisiae, the absence of Pif1 helicase induces the instability of G4-containing CEB1 minisatellite during leading strand but not lagging strand replication. We report that RPA and Pif1 cooperate to maintain CEB1 stability when the G4 forming strand is either on the leading or lagging strand templates. At the leading strand, RPA acts in the same pathway as Pif1 to maintain CEB1 stability. Consistent with this result, RPA coprecipitates with Pif1. This association between Pif1 and RPA is affected by the rfa1-D228Y mutation that lowers the affinity of RPA in particular for G-rich single-stranded DNA. At the lagging strand, in contrast to pif1 $\Delta$, the rfa1-D228Y mutation strongly increases the frequency of CEB1 rearrangements. We explain that Pif1 is dispensable at the lagging strand DNA by the ability of RPA by itself to prevent formation of stable G-rich secondary structures during lagging strand synthesis. Remarkably, overexpression of Pif1 rescues the instability of CEB1 at the lagging strand in the rfa1-D228Y mutant indicating that Pif1 can also act at the lagging strand. We show that the effects of the rfa1-D228Y (rpa1-D223Y in fission yeast) are conserved in Schizosaccharomyces pombe. Finally, we report that RNase $\mathrm{H} 1$ interacts in a DNAdependent manner with RPA in budding yeast, however overexpression of RNase $\mathrm{H1}$ does not rescue CEB1 instability observed in pif1 $\Delta$ and rfa1-D228Y mutants. Collectively these results add new insights about the general role of RPA in preventing formation of DNA secondary structures and in coordinating the action of factors aimed at resolving them.
\end{abstract}

doi: $10.15698 /$ cst2020.03.214

Received originally: 25.09.2018;

in revised form: 03.01.2020,

Accepted 07.01.2020,

Published 17.01.2020.

Keywords: RPA, Pif1, human minisatellite CEB1, G-rich structures, G-quadruplex.

\section{Abbreviations:}

Co-IP - Co-immunoprecipitation, $R P A$ - replication protein $A$, ssDNA - single-stranded DNA, WT-wild type.

\section{INTRODUCTION}

Replication protein A (RPA) is the major eukaryotic singlestranded DNA (ssDNA) binding protein that consists of 70 , 32 , and $14 \mathrm{kDa}$ subunits [1]. RPA plays a key role in coordinating DNA synthesis, repair, and DNA damage signalling through binding to single-stranded DNA (ssDNA) intermediates generated during these processes [2]. RPA primarily maintains sSDNA in an unfolded state through different binding modes that are characterized by the length of the interacting ssDNA [3]. Thus, RPA binds to ssDNA with high affinity preventing the formation of DNA secondary structures and annealing of homologous sequences [4]. Among its activities, RPA is involved in the maturation of Okazaki fragments by governing the sequential action of Dna2 and Fen1 [5]. During Pol $\delta$ synthesis most flaps generated on the lagging strand, by strand displacement, are normally cleaved by Fen1. However, a minor fraction escapes cleavage [6], and the 5' ssDNA flaps on Okazaki fragments get extended by Pif1, a 5' to $3^{\prime}$ helicase, to create substrates for RPA binding that inhibits Fen1's cleavage [5,7-11]. RPA is next displaced by Dna2 which cleaves the long flap, generating a short flap structure that undergoes cleavage via Fen1 [12-14].

Interestingly, it has been reported in vitro, that RPA binds to and unwinds G4 structures in a $5^{\prime}$ to $3^{\prime}$ direction [15]. G4 are polymorphic and consist of four-stranded 
structures formed at specific G-rich motifs within DNA, RNA, and into R loops, a RNA-DNA hybrid structure, that can eventually lead to genome instability [16-19]. The core of these structures is formed by a square arrangement of four guanines held together by Hoogsteen hydrogen bonds $[20,21]$. Under specific conditions, G4 structures are recognized by specific factors and their formation is controlled $[22,23]$. However, it has been shown that highly stable G4 structures impede fork progression. Hence, their unwinding by helicases is critical [17, 24, 25]. Many helicases are able to unwind $\mathrm{G} 4$ structures in vitro such as the RecQ helicases BLM, WRN and Sgs1 and other helicases such as Pif1, FANCJ or RTEL1 [24,26-30]. G4 structures are also targeted by additional proteins that protect them [23] or support the function of an helicase at G4 [22]. In budding yeast, unwinding of G4 is mainly performed by the Pif1 helicase [26]. Indeed, a particular example is the $1.8 \mathrm{~kb}$ G4-forming human minisatellite CEB1, a reporter of G4 formation and processing [31, 32]. In cells lacking Pif1, CEB1 is unstable when inserted into Saccharomyces cerevisiae genome, near an early origin of replication (ARS305). Instability of CEB1, which consists in 42 motifs of 39 nucleotides arranged as direct repeats, was correlated to the ability of the CEB1 motif to form G4 [31, 33, 34]. Surprisingly, in pif $1 \triangle$ cells $C E B 1$ was unstable only when the G-quadruplexforming strand was the leading strand template [33]. This result is apparently counterintuitive since ssDNA is mostly formed during the discontinuous synthesis of the lagging strand DNA [35] and because Pif1 has been reported to act at the lagging strand [36] and binds G4 structures located in the lagging strand [22, 37].

We have previously shown in fission yeast that the rpa1-D223Y mutation (rfa1-D228Y in budding yeast), that exhibits a reduced affinity for ssDNA, impaired lagging strand telomere replication and provoked accumulation of secondary structures [38]. Consistently, expression of ScPif1 rescued the phenotypes associated with the rpa1D223Y mutation [38]. These results suggested that rpa1D223Y cells accumulated G-rich structures at lagging strand telomeres that were resolved by the heterologous expression of ScPif1. In S. cerevisiae, RPA subunits are encoded by RFA1, RFA2, and RFA3 genes. The rfa1-D228Y mutant (rpa1-D223Y in fission yeast) also possesses a lower affinity for ssDNA and reduced ability in removing secondary structure from ssDNA $[39,40]$. In this study, we investigated the role of RPA in maintaining the stability of CEB1 when the G-quadruplex-forming strand is either on the leading or lagging strand template. Our results indicate that both RPA and Pif1 cooperate at the leading strand to maintain the stability of CEB1. Consistent with this hypothesis, RPA co-precipitates with Pif1. In contrast to Pif1, RPA is also required to stabilize CEB1 when the G-quadruplex-forming strand is the lagging strand. However, under a situation that compromises RPA binding to ssDNA, overexpression of Pif1 rescued lagging-CEB1 instability, suggesting that Pif1 can unwind G4 at the lagging strand. Interestingly, Mms1 which binds to G-rich/G4 regions and supports the binding of Pif1, is not required to maintain the stability of CEB1. Based on these data we propose a model in which RPA facilitates Pif1 action at the leading strand DNA to unwind G4 while enriched RPA at the lagging strand DNA prevents by itself formation of stable G4, explaining why Pif1 is dispensable. We extended the role of RPA in preventing non-templated DNA singlestranded structure by showing that RPA interacts with RNAse $\mathrm{H} 1$ in a DNA-dependent manner in S. cerevisiae, as previously reported in human cells [41]. However, we found that overexpressing RNAse $\mathrm{H} 1$ did not restore CEB1 stability in both pif1 $\triangle$ and $r f a 1-D 228 Y$ mutants, suggesting that CEB1 instability is not due to R-Loop formation arising during transcription.

\section{RESULTS}

The rfa1-D228Y mutation affects both the leading-CEB1 and lagging-CEB1

As mentioned above, the G-rich minisatellite CEB1 can be considered as a reporter of G4 formation and processing $[31,32]$. We used strains previously constructed in A. Nicolas' laboratory in which the $1.8 \mathrm{~kb}$ CEB1 is inserted in both directions at $2.1 \mathrm{~kb}$ of ARS305 and $32.6 \mathrm{~kb}$ away from ARS306 allowing to primarily replicate CEB1 only from the proximal ARS305 origin (Figure 1). Depending on the orientation of CEB1 insertion, the G4-forming strand will be replicated by the leading or the lagging machinery [33]. We
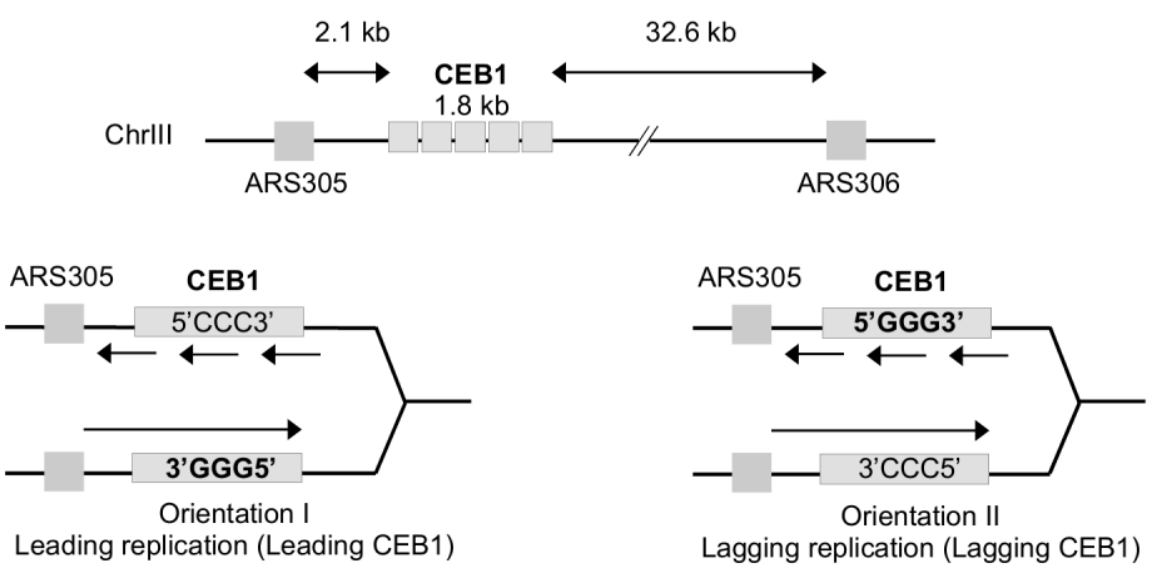

FIGURE 1: Map of the CEB1 insertion within chromosome III at $2.1 \mathrm{~kb}$ from the ARS305. In orientation I, the G-quadruplex-forming strand is the template of the leading polymerase (leading CEB1). In orientation II, the G-quadruplex-forming strand is the template of the lagging polymerase (lagging CEB1). 
A
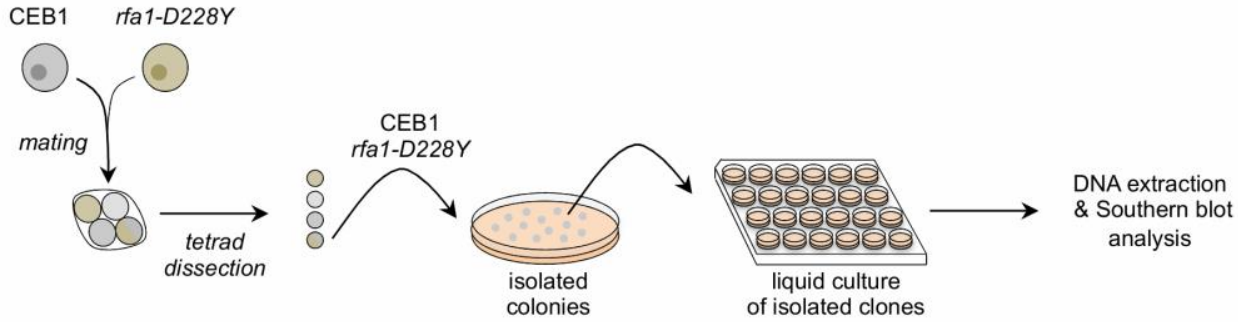

B

WT

pif1 $\Delta$

Ifa1-D228Y

pif1 Ifa1-D228Y

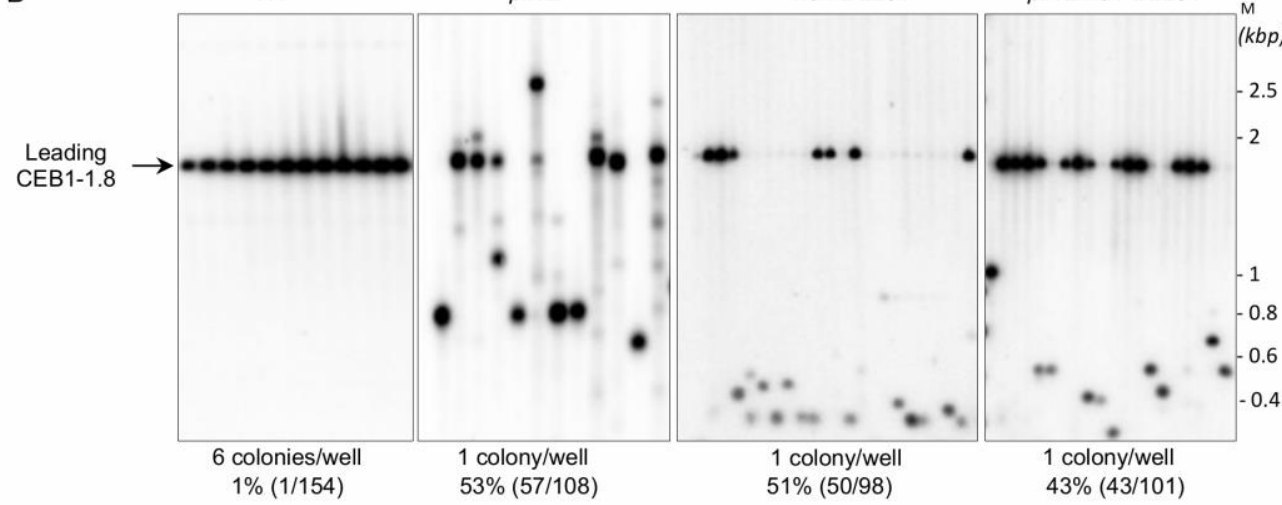

C

WT

pif1ム

rfa1-D228Y

pif1 1 ra1-D228Y

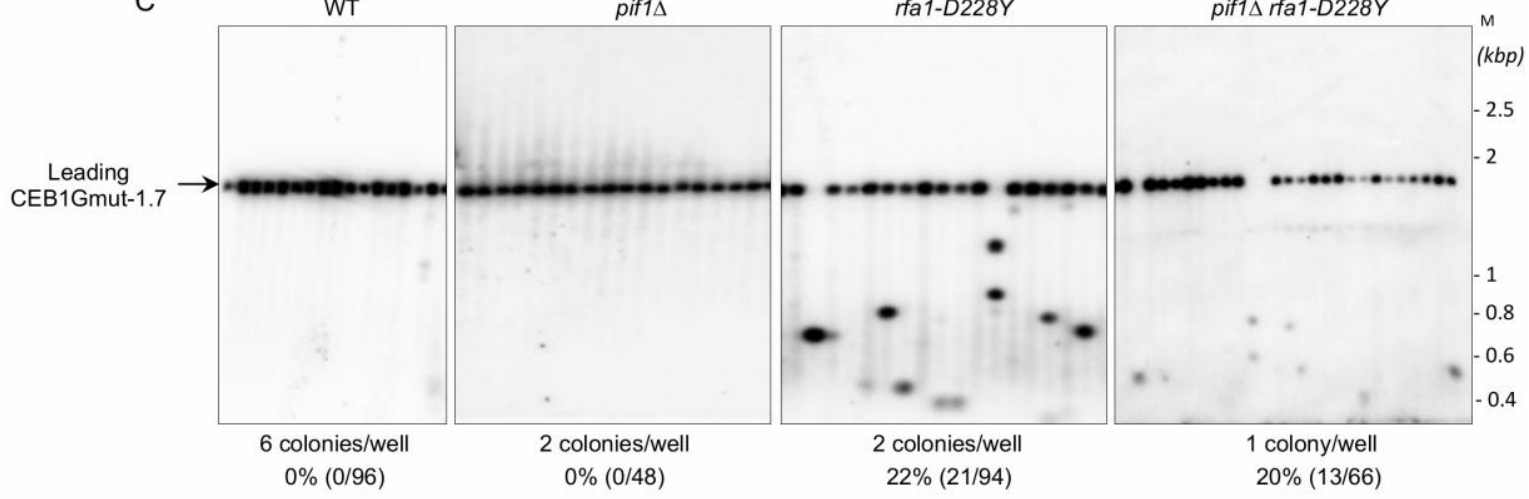

D WT

pif1 $1 \Delta$

rfa1-D228Y

pif1 rfa1-D228Y

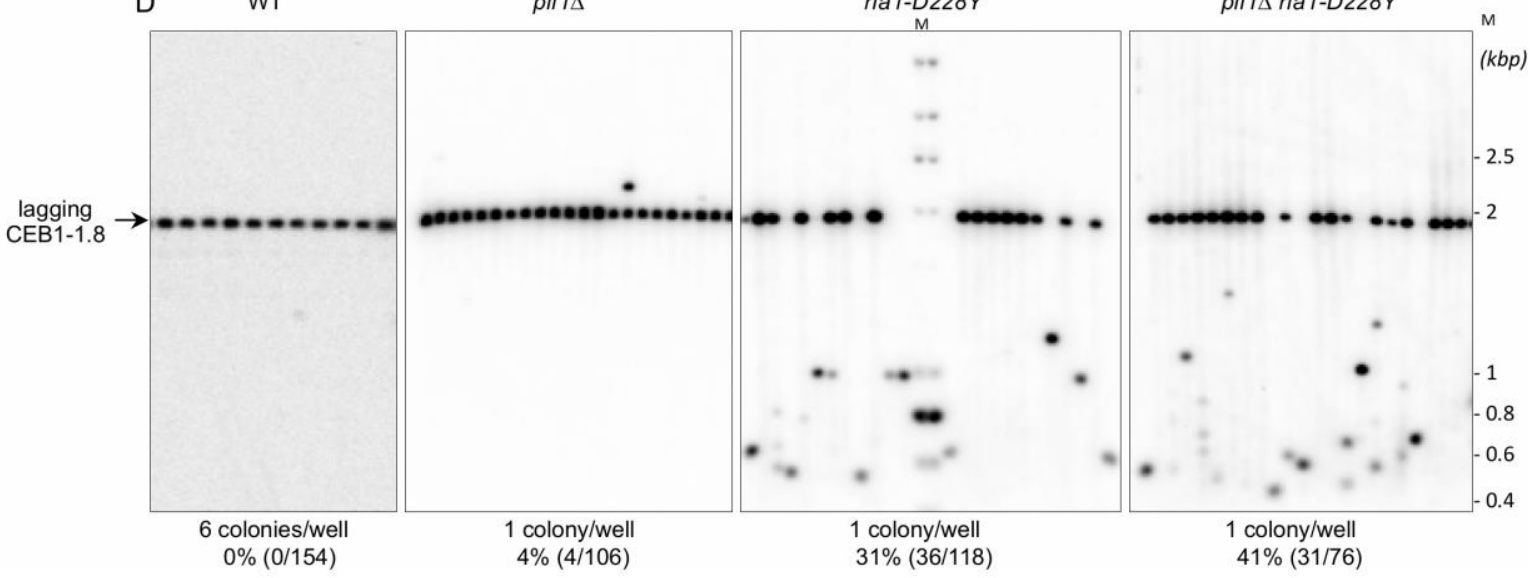

FIGURE 2: RPA is required to stabilize CEB1. (A) Experimental scheme. Yeast strains of interest are mated and the resulting diploid sporulated and dissected. After identification of spore-colonies of interest, the spore-colony is plated on media to obtain isolated colonies. Individual colonies are placed in liquid culture until stationary growth phase. Genomic DNAs are extracted and analysed by Southern blot. (B) RPA is required to stabilize CEB1 when the G4-forming strand is replicated by the leading polymerase. Genomic DNAs from yeast cells bearing the leading-CEB1 were digested by Apal and Xhol, and southern blotted. Membranes were hybridized with the CEB1-0.6 probe. (C) Genomic DNAs from yeast cells containing the leading-CEB1Gmut-1.7 were digested by Apal and Sacll, and southern blotted. The membranes were hybridized with the CEB1Gmut-1.7 probe. (D) In contrast to Pif1, RPA is required to stabilize CEB1 when the G4-forming strand is replicated by the lagging polymerase. Genomic DNAs from yeast cells bearing the lagging-CEB1 were digested by Apal and Ncol, and southern blotted. Membranes were hybridized with the CEB1-0.6 probe. M: ladder DNA serving as size standard (kbp). The number of colonies analysed per well, the percentage of rearrangement frequencies, and the total numbers of colonies are indicated in Table 1. 
will name leading-CEB1 or lagging-CEB1 to indicate the machinery that replicates the G4-forming strand. Previous results demonstrated that the helicase activity of Pif1 was required to stabilize the leading-CEB1 but that Pif1 was dispensable for the stability of the lagging-CEB1 suggesting the existence of different mechanisms to resolve $\mathrm{G} 4$ when the G4-forming strand is replicated by the leading or the lagging machinery [33].

We thought to test the role of RPA in the CEB1 stability by determining the effect of the rfa1-D228Y mutant whose DNA-binding activity, in particular to G-rich ssDNA, is compromised $[38,39]$. We used an experimental scheme (Figure 2A) adapted from Lopes et al. [33]. Briefly, tetrads obtained from the sporulation of heterozygous diploids were dissected. Spore colonies carrying the appropriated mutation were then resuspended in water and streaked on YPD plates in order to obtain about 200 isolated colonies. Colonies were inoculated in YPD liquid cultures, grown to saturation, and genomic DNAs were prepared from the liquid cultures. The size of CEB1 was monitored by Southern blot. We calculated the frequency of instability by monitoring CEB1 size variations (contractions and expansions). We considered that CEB1 was unstable when the intensity of the band(s) was superior to the one of the parental band. We therefore partially discriminated between early or late events of CEB1 rearrangements.

We first analysed the stability of the leading-CEB1 (orientation I) in wild type (WT), pif1 $\Delta$, rfa1-D228Y, and pif1 $\Delta$ rfa1-D228Y cells (Figure 2B). As shown in representative gels and as previously reported [31], the leading-CEB1 was stable in the WT strain but extremely unstable in the pif1 $\Delta$ mutant (53\% of rearrangements) (Figure 2B, Table 1). In most cases, we obtained contractions except in one case (Figure 2B, second panel). In the rfa-1D228Y mutant, leading-CEB1 was also unstable (51\% of rearranged colonies). Interestingly, the double mutant pif1 $\mathrm{rfa1}-\mathrm{D} 228 \mathrm{Y}$ exhibited a level of instability in a similar range of the two single mutants (43\%), suggesting that RPA and Pif1 could act in similar pathways to unwind G4 and stabilize CEB1 when the G4-forming strand is replicated by the leading polymerase (Figure 2B).

We next analysed the effect of pif1 $\Delta$, rfa1-D228Y, and pif1 1 rfa1-D228Y on the stability of CEB1-Gmut, a version of CEB1 mutated for its G-quadruplex-forming sequences $[31,32]$. The CEB1-Gmut was inserted at the same location and in the same orientation as the leading-CEB1. As reported, the CEB1-Gmut was stable in pif1 $\triangle$ cells [33]. In the rfa1-D228Y and pif1 rfa1-D228Y mutants, the \% of CEB1Gmut rearrangements dropped to $22 \%$ and $20 \%$, respectively (Figure $\mathbf{2 C}$ ). We concluded that the high leadingCEB1 instability observed in the rfa1-D228Y mutant partly relies on its ability to form $\mathrm{G} 4$. However, the residual CEB1Gmut instability suggests that the rfa1-D228Y mutation affects the replication of the 42 repeated motifs of CEB1mut irrespectively of the presence of G4.

We next examined the stability of the lagging-CEB1 (orientation II) in the same mutants as above. As reported [33], deleting PIF1 had no effect on the stability of the lagging-CEB1 (Figure 2D). In contrast, the frequency of rear- rangements of the lagging-CEB1 was clearly increased in the rfa1-D228Y mutant (31\%). These results suggest that when G4-forming sequences are localized at the lagging strand, RPA prevents the instability of CEB1. They are consistent with the known enrichment of RPA at the lagging strand [35]. Interestingly, despite the fact that pif1 $\Delta$ did not affect the stability of the lagging-CEB1, combining pif1 $\Delta$ with rfa1-D228Y, slightly aggravated the CEB1 instability phenotype of the single rfa1-D228Y mutant (41\%). This result suggests that Pif1 can be active at the laggingCEB1.

\section{Overexpression of Pif1 in rfa1-D228Y cells rescues lagging- CEB1 instability}

To further understand the functional interaction between RPA and Pif1 in CEB1 instability, we overexpressed Pif1 in rfa1-D228Y cells bearing leading-CEB1 or lagging-CEB1. Because strong Pif1 overexpression impairs cell viability [42], we used a low-copy centromeric plasmid in which the nuclear form of Pif1 is under the control of the GAL1 promoter [43]. We first noticed that Pif1 overexpression was slightly deleterious in rfa1-D228Y cells (Figure 3A). To estimate Pif1 overexpression we measured the expression of PIF1 by reverse transcription followed by quantitative PCR. Our data indicate that PIF1 is overexpressed in a similar level in WT and rfa1-D228Y cells expressing PIF1 under the control of the GAL1 promoter (Figure 3B, left and $3 \mathbf{C}$, left). Instability of both leading- and lagging-CEB1 was tested in rfa1-D228Y cells grown in galactose (SGal) allowing the overexpression of Pif1. We found that Pif1 overexpression had only a modest effect on leading-CEB1 stability in rfa1D228Y cells while it had no effect in WT cells (Figure 3B, right). Surprisingly, Pif1 overexpression completely rescued the instability of lagging-CEB1 in rfa1-D228Y cells (Figure 3C, right). We concluded that when the G4-forming strand was replicated by the lagging machinery in cells in which the sSDNA binding activity of RPA is compromised, overexpressed Pif1 could act at the lagging strand to unwind G4.

\section{The rfa1-D228Y mutation affects the interaction between Pif1 and RPA}

Our results indicate that RPA and Pif1 could cooperate to stabilize the leading-CEB1 and the lagging-CEB1. This prompted us to test whether Pif1 interacts with RPA. Pif1myc was immunoprecipitated with anti-Myc antibody and tested for the presence of RPA with a polyclonal antibody directed against Rfa1 or against Rfa2 (Figure 4). Pif1-myc was efficiently co-immunoprecipitated with both Rfa1 and Rfa2 but to a lesser extend with rfa1-D228Y. To address whether the robust Co-immunoprecipitations (Co-IP) of Pif1-myc with Rfa1 and Rfa2 were dependent on the presence of DNA, DNase1 was added in the lysate. As shown in Figure 4, DNA digestion affected the Co-IP suggesting that the presence of DNA is required for the robust coprecipitation of Pif1 and RPA. These results suggest that the robust association of Pif1 with RPA relies on specific structures on the DNA. We think that the interaction between Pif1 and RPA is not only due to unspecific interactions mediated by DNA since the interaction between Pif1 
A

Leading CEB1

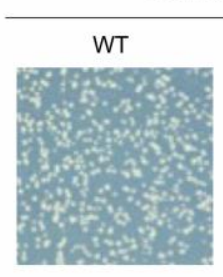

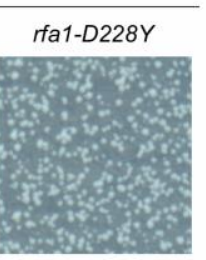

Lagging CEB1

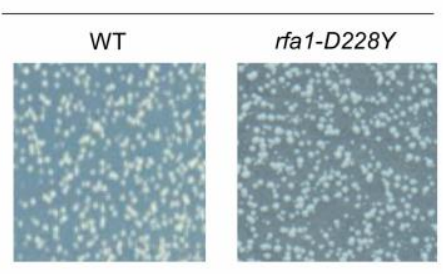

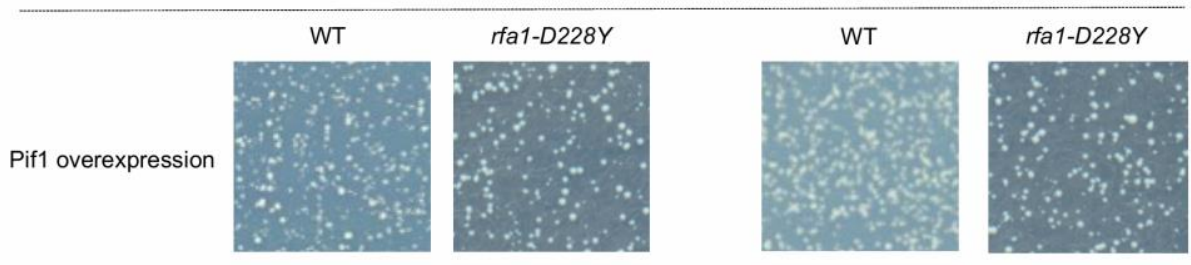

B
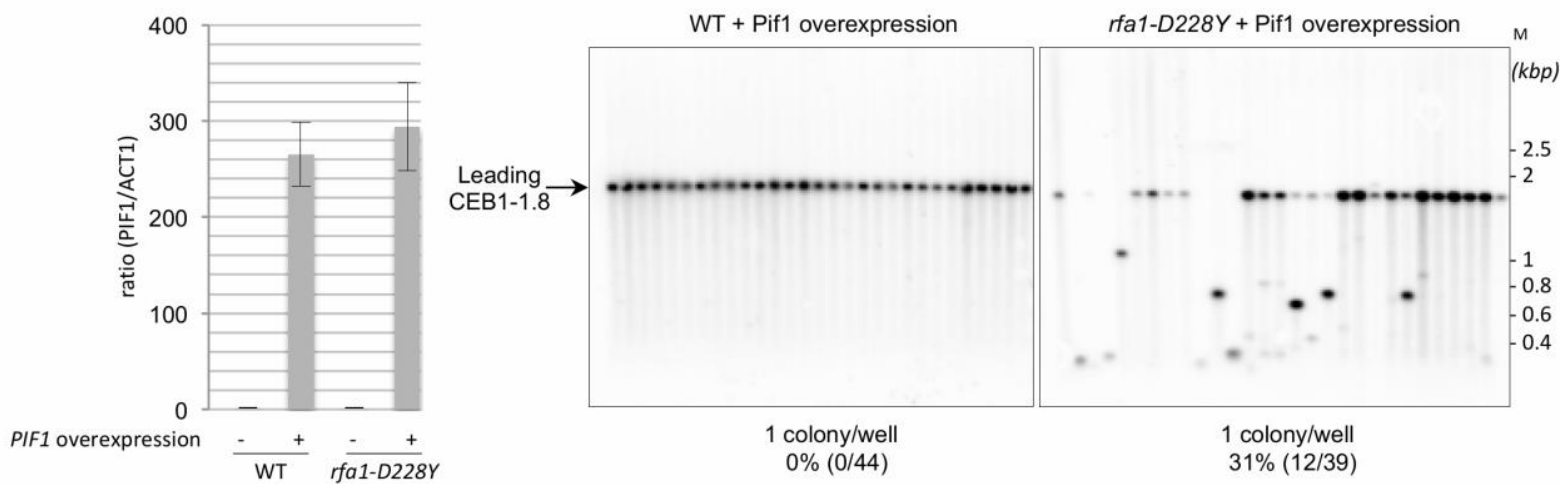

C
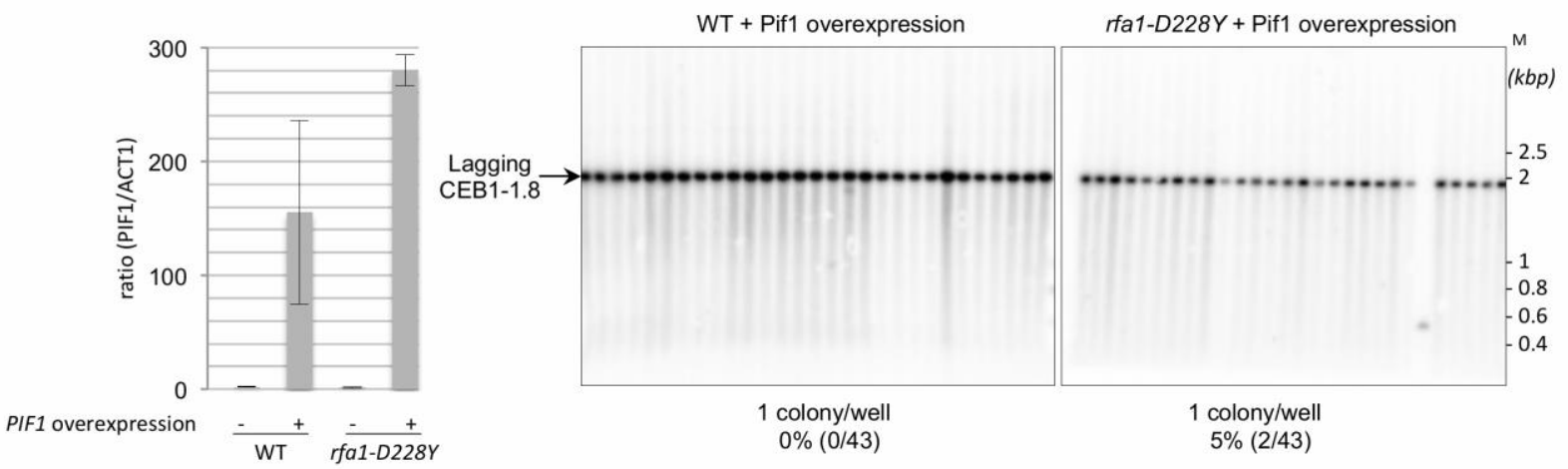

FIGURE 3: Pif1 overexpression rescues lagging-CEB1 instability in the rfa1-D228Y mutant. (A) Colonies containing the leading- or lagging-CEB1, and overexpressing Pif1 (Pif1 overexpression) or not (Empty vector) were plated on galactose medium and subsequently incubated at $30^{\circ} \mathrm{C}$. (B) Left: $\mathrm{CDNA}$ was prepared from the indicated strains. The ration of PIF1 transcript to that of ACT1 was determined using qPCR. (-): Empty vector, (+): Pif1 overexpression. Right, genomic DNAs from wild-type (WT) or rfa1-D228Y cells overexpressing Pif1 (Pif1 overexpression) and containing the leading-CEB1 were treated as in Figure 2. M: ladder DNA. (C) Left: cDNA was prepared from the indicated strains. The ration of PIF1 transcript to that of $A C T 1$ was determined using qPCR. (-): Empty vector, (+): Pif1 overexpression. Right, genomic DNAs from wild-type (WT) or rfa1-D228Y cells overexpressing Pif1 (Pif1 overexpression) and containing the lagging-CEB1 were treated as in Figure 2. M: ladder DNA. Yeast strains were grown in medium containing galactose (2\%). The number of colonies analysed per well, the percentage of rearrangement frequencies, and the total numbers of colonies are indicated in Table 1. 


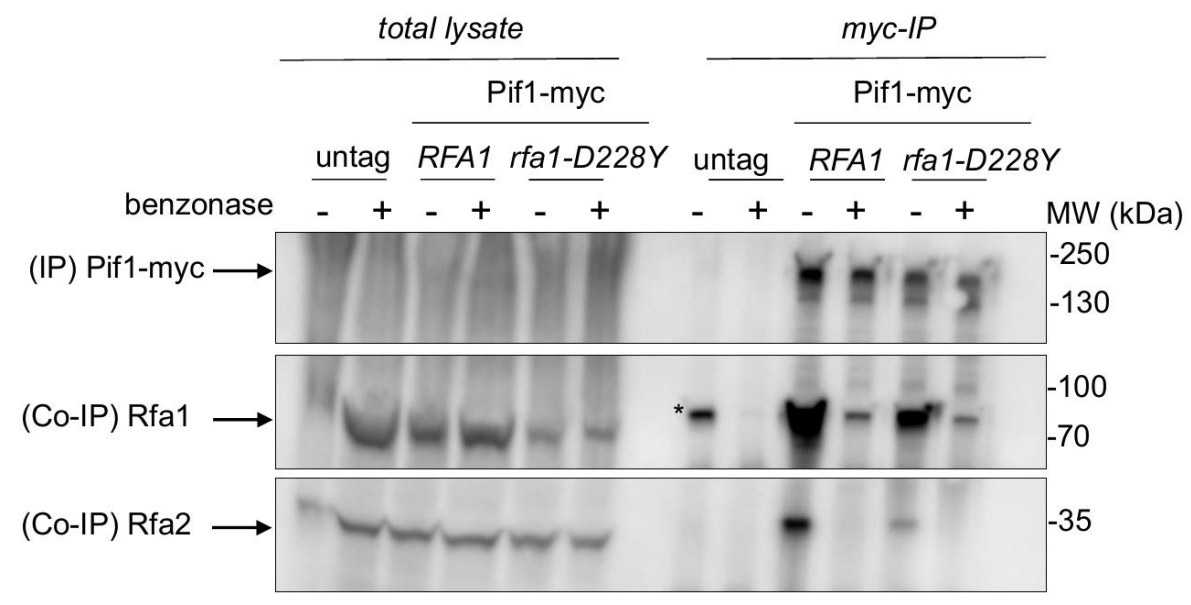

FIGURE 4: The rfa1-D228Y mutation affects the interaction between Pif1 and RPA. Coimmunoprecipitation experiments were performed in triplicate. Pif1myc is immunoprecipitated with an anti-Myc antibody (9E10). The presence of RPA in the Pif1-myc IP is monitored with an anti-Rfa1 or an anti-Rfa2 antibody. (+): cell extracts treated with benzonase. The asterisk $\left(^{*}\right)$ indicates a nonspecific band. MW: molecular weight.

and $r f a 1-228 Y$ is lost despite the fact that the rfa1-D228Y still binds to DNA, although with lower affinity [38].

\section{Mms1 is not required to maintain the leading-CEB1 and the lagging-CEB1 stability}

Mms1 supports Pif1 helicase binding to G4 structures [22]. We thus analysed the importance of Mms1 in CEB1 stability. We found that in $m m s 1 \Delta$ cells, leading-CEB1 and lagging-CEB1 were rather stable, showing $0 \%(0 / 116)$ and $3 \%$ (3/116) of rearrangements, respectively (Figure 5A, Table 1). These results show that Mms1 is not required for the stability of both leading-CEB1 and lagging-CEB1. They suggest that CEB1 minisatellites are not targeted by Mms1. Moreover, they indicate that Mms1 does not support Pif1 function at CEB1 and, together with our previous results showing that Pif1 and RPA interact, suggest that RPA could contribute to the recruitment of Pif1 to CEB1, likely by directly recruiting Pif1.

\section{Rtt105 is required to stabilize both the leading-CEB1 and lagging-CEB1}

Rtt105 functions as an RPA chaperone that escorts RPA to the nucleus and facilitates RPA loading onto ssDNA [44]. Consequently, RTT105 inactivation reduces the association of RPA with ssDNA generated during DNA transactions and affects multiple RPA functions [44, 45]. We examined the importance of Rtt105 on the stability of the leading-CEB1 and lagging-CEB1. We found that CEB1 is extremely unstable in rtt105 cells. Remarkably, we found that the frequency of rearrangements reaches $100 \%$ in both leadingand lagging-CEB1 (Figure 5B, Table 1). These results reveal the importance of RTT105 in promoting the replication of G4-forming CEB1 minisatellite during leading and lagging strand synthesis and more generally, to maintain genome stability. They confirm our previous results obtained with rfa1-D228Y mutant indicating that RPA is crucial to remove G-rich structures at both leading and lagging strand.
RNase H1 interacts with RPA but its overexpression doesn't rescue CEB1 instability in the absence of Pif1 or reduced levels of RPA

Transcription by RNA polymerase can form a threestranded structure called R-loop [46], which can facilitate or stabilize secondary structure formation in the exposed ssDNA [16, 47, 48]. Thus, G4 structures may also result from exposure of the $\mathrm{G} 4$ forming strand by formation on the other strand of RNA:DNA hybrids. This may create a complex structure involving G4 DNA on one strand and a RNA:DNA hybrid on the other strand [49]. Interestingly, Pif1 has been proposed to have a patrolling role that removes any G4 or RNA/DNA structure [50]. Indeed, Pif1 regulates $\mathrm{R}$-loop formation at specific genomic loci [51] and potentially complements RNAse $\mathrm{H}$ for R-loop resolution [52]. On the other side, systematic analysis of protein complexes in $S$. cerevisiae have shown that RPA interacts with RNase $\mathrm{H} 1$ [53]. Finally, RPA was recently proposed to act as a sensor of R-loop in human cells and to recruit and stimulate RNase $\mathrm{H} 1$ to counteract R-loops [41]. To further document the potential cooperation of RPA with Pif1, we first tested whether RPA interacts with RNase $\mathrm{H} 1$ in a DNAdependent manner in budding yeast. Expression of HARnh1 was induced by the addition of galactose and its interaction with Rfa1 was probed by Co-IP. The results shown in Figure 6 indicate that Rnh1 interacts with RPA in a way that is stimulated by the presence of DNA, thereby extending the results of Gavin et al. [53] and Nguyen et al. [41]. Interestingly, we found that the rfa1-D228Y mutant had a less efficient ability to bind Rnh1 (Figure 6). These results suggest that the leading-CEB1 instability observed on the leading strand in absence of Pif 1 and by reducing the association of RPA with ssDNA ( $r f a 1-D 228 Y$ and $r t t 105 \Delta$ mutants) could be the consequence of the presence of RLoops. To determine if $\mathrm{R}$-loops were responsible of CEB1 instability we analysed the stability of the leading-CEB1

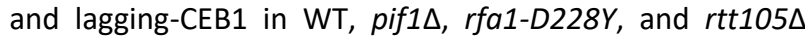
cells overexpressing RNase $\mathrm{H} 1$, the enzyme responsible to resolve R-loops. We found that RNase $\mathrm{H} 1$ overexpression did not affect CEB1 stability in WT cells, and did not rescue

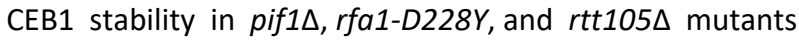


A
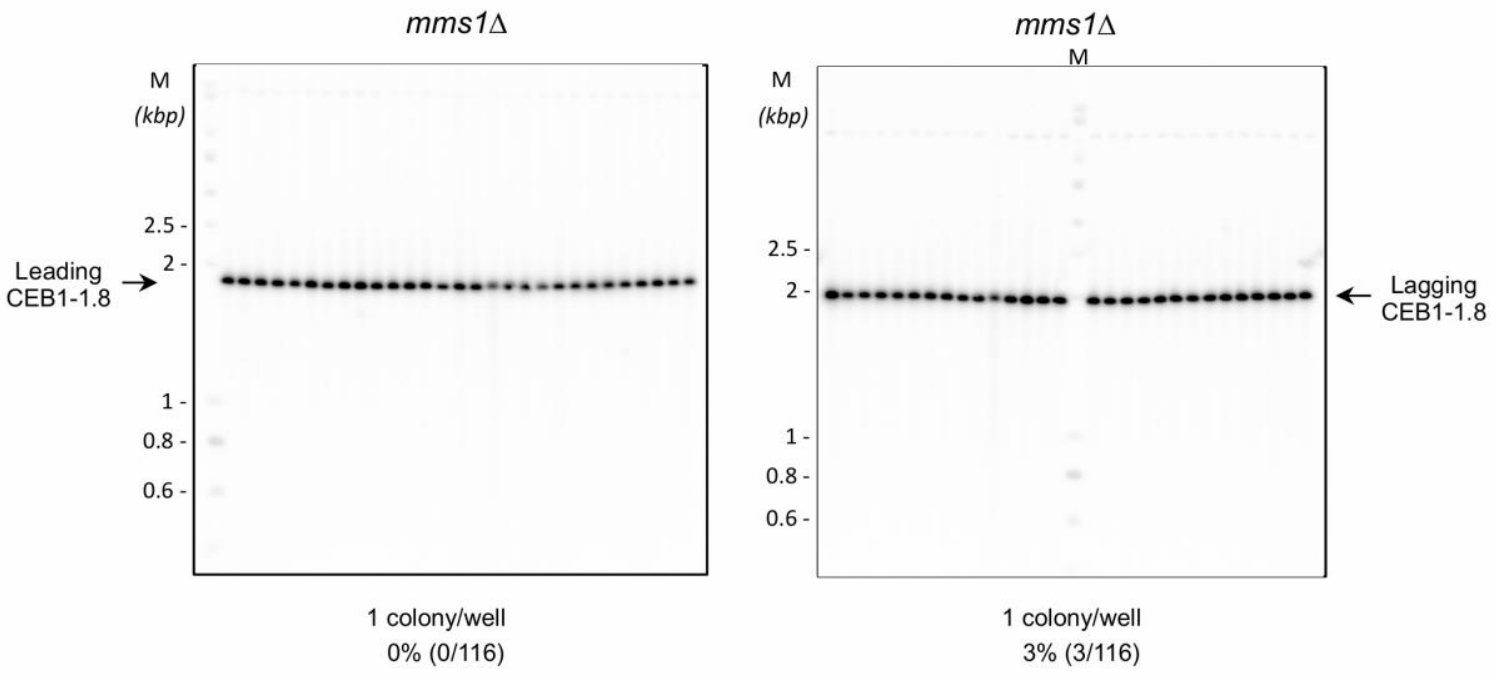

B

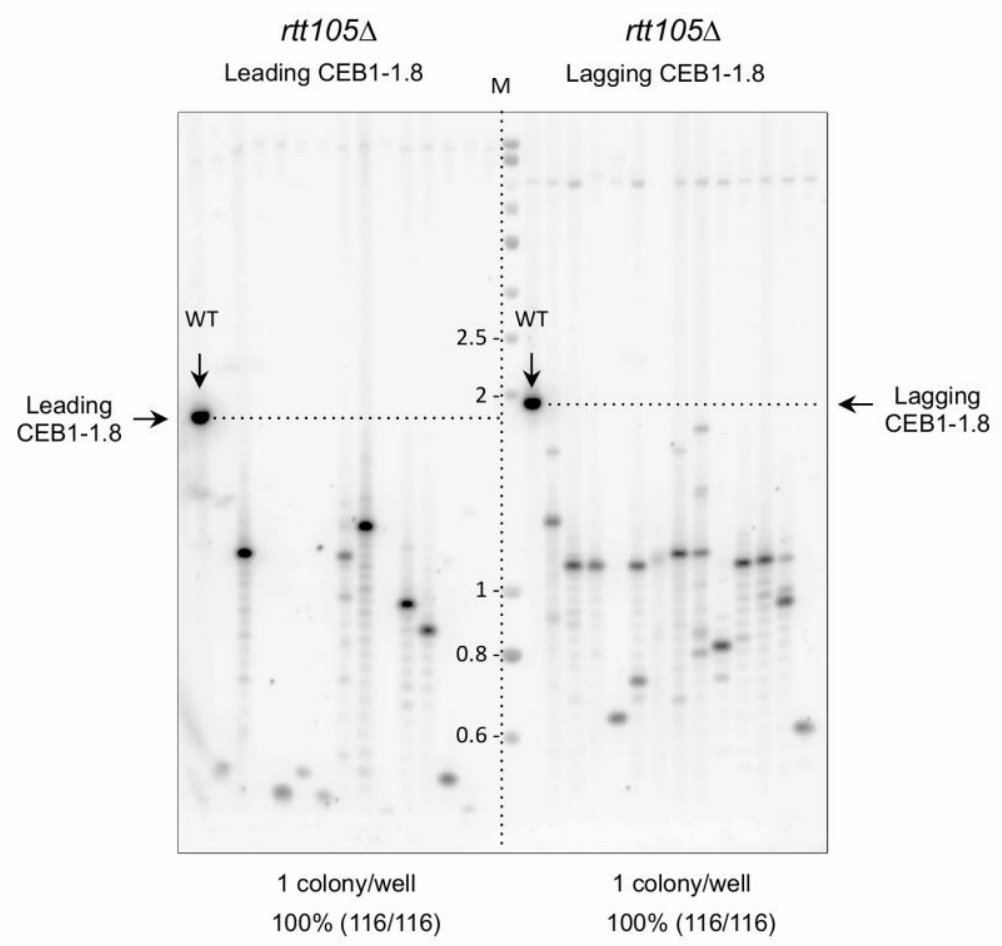

FIGURE 5: Importance of Mms1 and Rtt105 on CEB1 stability. (A) Mms1 is not required to stabilize CEB1. Left, genomic DNAs from mms1 14 yeast cells bearing the leading-CEB1 were digested by $A p a l$ and Xhol and southern blotted. Right, genomic DNAs from $m m s 1 \Delta$ yeast cells bearing the lagging-CEB1 were digested by Apal and Ncol and southern blotted. Membranes were hybridized with the CEB1-0.6 probe. (B) Rtt105 is required to stabilize both leading-CEB1 and lagging-CEB1. Left, genomic DNAs from rtt105 yeast cells bearing the leading-CEB1 were digested by Apal and Xhol and southern blotted. Right, genomic DNAs from rtt105 yeast cells bearing the lagging-CEB1 were digested by Apal and Ncol and southern blotted. Membranes were hybridized with the CEB1-0.6 probe. WT: wild-type genomic DNA. The arrows show the position of stable leading-CEB1 (left) and stable lagging-CEB1 (right). The horizontal dashed lines indicate the theoretical position of stable CEB1. M: ladder DNA serving as size standard (kbp). The number of colonies analysed per well, the percentage of rearrangement frequencies, and the total numbers of colonies are indicated in Table 1. 


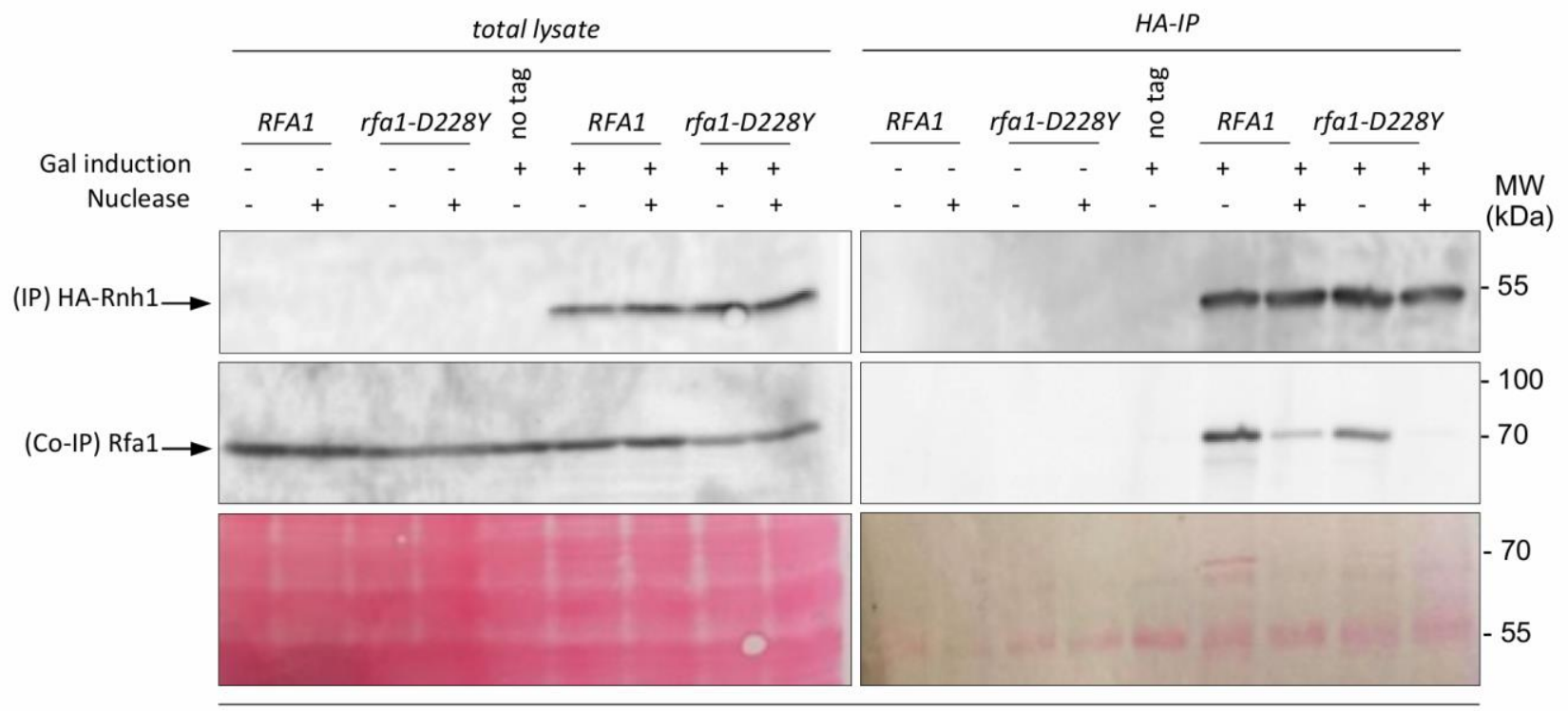

Gal-HA-Rnh1

FIGURE 6: Rnh1 interacts with RPA. Co-immunoprecipitation experiments were performed in triplicate. HA-Rnh1 has been overexpressed by galactose addition and immunoprecipitated with an anti-HA antibody. The presence of RPA in the HA-Rnh1 IP is monitored with an anti-Rfa1 antibody. Cell extracts treated with nuclease (Thermofisher) are indicated (+). Yeast strains were grown in medium containing $2 \%$ glucose $(-)$ or $2 \%$ galactose $(+)$ when mentioned. Total proteins on the membrane were stained with Ponceau $\mathrm{S}$ as a loading control (Bottom). MW: molecular weight.

(Table 1). Taken together, our data suggest that the instability observed in absence of Pif1 and reduced levels of RPA is not primarily related to $\mathrm{R}$-loop formation.

\section{The rpa1-D223Y mutation affects the stability of CEB25- L1T in fission yeast}

Because the rpa1-D223Y mutation impaired replication of the G-rich lagging strand telomere in Schizosaccharomyces pombe, we investigated the stability of CEB in fission yeast. We took advantage of the minisatellite CEB25-L1T which contains 14 repeats of a 44 nucleotides-sequence $(0.62 \mathrm{~kb})$ that form a stable $\mathrm{G} 4[34,54]$. In this L1T version, the loop of the repeated sequence of CEB25 has been reduced to one thymine nucleotide. The shortening of the loop increases its thermal stability in correlation with the in vivo instability [54]. As a control the CEB25-L1T-G12T has been used in which the guanine at the $12^{\text {th }}$ position of the repeated sequence has been mutated into thymine, preventing the formation of G4. We introduced the CEB25-L1T and CEB25-L1T-G12T in both orientations into the genome of yeast cells at the leu1 locus at chromosome 2. The leu1 locus is located in between ARS-II-1964 and ARS-II-1983 at a distance of $14 \mathrm{~kb}$ and $11 \mathrm{~kb}$ from CEB25, respectively (Figure 7A). These two ARS have a relatively low firing efficiency, $32 \%$ and $13 \%$, respectively [55]. We monitored in fission yeast the instability of CEB25 in both orientations although this genomic context does not allow to clearly distinguish whether the G4-forming sequence is replicated by the leading or the lagging replication machinery.

As depicted in Figure 2A, the size of CEB25-L1T was monitored by Southern blot and frequency of instability was calculated in both WT and rpa1-D223Y strains. The level of instability of CEB25-L1T was $14 \%$ and $20 \%$ in the WT (Figure 7B, left). In the rpa1-D223Y mutant, this level increased up to $62 \%$ and $74 \%$, respectively (Figure 7B, right). Expectedly, the instability of CEB25-L1T-G12T that cannot form $\mathrm{G} 4$ was reduced to $4 \%$ for leading replication and $17 \%$ for lagging replication (Figure $7 \mathrm{C}$ ). These results clearly show that like in budding yeast the RPA complex plays an important role in the replication and stability of G4-forming sequences. Furthermore, the fact that fission yeast lacks a functional homolog of Pif1 helicase, the Pfh1 helicase behaving more closely like the budding yeast Rrm3 helicase [56], may account for the prominent role of RPA complex in $\mathrm{G} 4$ unwinding in fission yeast.

\section{DISCUSSION}

In S. cerevisiae, Pif1 helicase processes G-rich secondary structures thereby preventing deleterious events that may lead to DNA breaks $[33,38,57]$. In vitro, Pif1 binds tightly to G4 structures and unwinds them very efficiently $[26,58]$.

Previous studies demonstrated that Pif1 prevents the formation of G-quadruplex-dependent CEB1 internal rearrangements during leading strand, but not lagging strand replication [33]. Here, we disclose that RPA cooperates with Pif1 to remove G-quadruplex structures at both leading and lagging strand. We found that the rfa1-D228Y mutation increases the frequency of CEB1 rearrangements when the G-quadruplex forming strand is replicated by the leading polymerase. The level of rearrangements is similar to the level observed in pif1 $\Delta$ cells. We report that mutating G-quadruplex-forming sequences strongly decreases 
TABLE 1. Rearrangement frequencies of CEB1 placed near ARS305 in both orientations in WT and mutant strains.

\begin{tabular}{|c|c|c|c|}
\hline Minisatellite & Genotype & Orientation I & Orientation II \\
\hline \multirow[t]{12}{*}{ CEB1-1.8 } & WT & $1 / 154(1 \%)$ & $0 / 154(0 \%)$ \\
\hline & pif1 $\Delta$ & $57 / 108(53 \%)$ & $4 / 106$ (4\%) \\
\hline & rfa1-D228Y & $50 / 98(51 \%)$ & $36 / 118(31 \%)$ \\
\hline & pif1 $\Delta$ rfa1-D228Y & $43 / 101(43 \%)$ & $31 / 76(41 \%)$ \\
\hline & WT GAL::PIF1 & $0 / 44(0 \%)$ & $0 / 43(0 \%)$ \\
\hline & rfa1-D228Y GAL::PIF1 & $12 / 39(31 \%)$ & $2 / 43(5 \%)$ \\
\hline & $m m s 1 \Delta$ & $0 / 116(0 \%)$ & $3 / 116(3 \%)$ \\
\hline & $r t t 105 \Delta$ & $116 / 116(100 \%)$ & $116 / 116(100 \%)$ \\
\hline & WT GAL::RNH1 & $1 / 58(2 \%)$ & $0 / 58(0 \%)$ \\
\hline & pif1 $\triangle$ GAL::RNH1 & $30 / 58(52 \%)$ & ND \\
\hline & rtt105 GAL::RNH1 & $58 / 58(100 \%)$ & $58 / 58$ (100\%) \\
\hline & rfa1-D228Y GAL::RNH1 & $39 / 87(45 \%)$ & $29 / 87(33 \%)$ \\
\hline \multirow[t]{4}{*}{ CEB1-Gmut-1.7 } & WT & $0 / 96(0 \%)$ & ND \\
\hline & pif1 $1 \Delta$ & $0 / 48(0 \%)$ & ND \\
\hline & $r f a 1-D 228 Y$ & $21 / 94(22 \%)$ & ND \\
\hline & pif1 1 rfa1-D228Y & $13 / 66(20 \%)$ & ND \\
\hline
\end{tabular}

ND - not determined.

the instability of CEB1 in rfa1-D228Y while it totally abolishes the instability of CEB1 in pif1 $1 \Delta$ cells. Contrary to pif1 cells, in rfa1-D228Y cells the CEB1 instability does not completely rely on the G-quadruplex-forming sequence of CEB1, indicating that the instability arising in this mutant partially results from G-quadruplex-independent ssDNA-containing secondary structures. We obtained similar results with the double pif1 $r$ ra1-D228Y mutant. Consistent with these results, Pif1 overexpression suppresses the instability of rfa1-D228Y mutant in the same proportion as G-mutated CEB1 motif. We therefore propose that RPA cooperates with Pif1 to resolved G-quadruplexes during leading strand replication.

In contrast to Pif1, which is not required for CEB1 stability during lagging strand replication, we found that RPA has a prominence in maintaining lagging CEB1 stability. This finding points out the differential behaviour of the pif1 $\Delta$ and $r f a 1-D 228 Y$ mutations according to the direction of replication. However, our results indicate that Pif1 overexpression drastically reduced the instability observed at the lagging strand in the rfa1-D228Y mutant whose ssDNA binding activity of RPA is compromised, in particular to G-rich regions [38]. Finally, we report that Mms1, which binds G4-structures and aids Pif1 binding to these structures is not required for CEB1 stability at both leading and lagging strands. In contrast Rtt105 that promotes RPA nuclear import, and RPA-ssDNA complex formation at replication forks is required to stabilize CEB1 inserted in both orientations. Collectively these results indicate that Pif1 and
RPA cooperate to remove G-quadruplex structures at both leading and lagging strand (Figure 8).

Several interpretations can be invoked to explain the difference in CEB1 stability observed in pif1 cells during leading and lagging replication. It is possible that RPA recruits Pif1. We think that the difference in Pif1 requirement at leading and lagging strands is related to RPA function. At the leading strand, both Pif1 helicase and RPA are required. However, our results showing that mutated CEB1 (which are no longer able to form G4-strutures) and Pif1 overexpression both only partially rescue instability in rfa1D228Y cells reveal higher requirement of Pif1 compared to RPA to maintain CEB1 stability at the leading strand. Interestingly the roles are inverted between RPA and Pif1 at the lagging strand. Because contrary to the leading strand the lagging strand contains longer stretch of SsDNA between elongating Okazaki fragments, RPA is present at a higher concentration at the lagging strand [59]. Consequently, by preventing formation/folding of G-quadruplex structures at the exposed ssDNA, RPA is likely to directly contribute to CEB1 stability independently of Pif1 at the lagging strand. Because rfa1-D228Y possesses a lower affinity for ssDNA and a reduced ability in preventing and removing secondary structures from ssDNA, in rfa1-D228Y cells CEB1 becomes unstable. In that situation Pif1, which is not initially required to remove G-quadruplex at the lagging strand, is now required. Our results showing that the Pif1 overexpression almost fully rescues the stability of CEB1 in rfa1D228Y mutant at the lagging strand suggest that CEB1-Gquadruplexes are formed in this mutant and can be pro- 
A

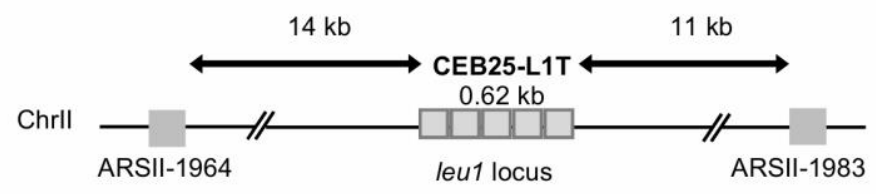

B
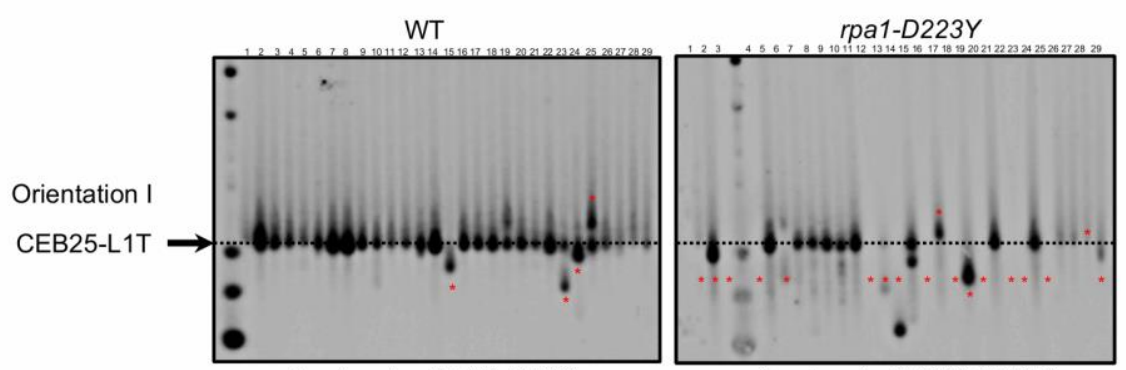

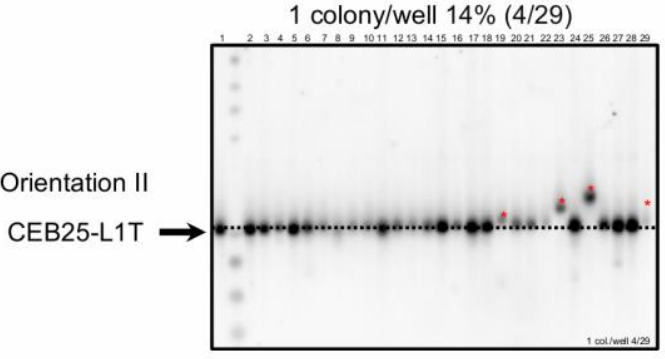

1 colony/well $20 \%(20 / 99)$

C

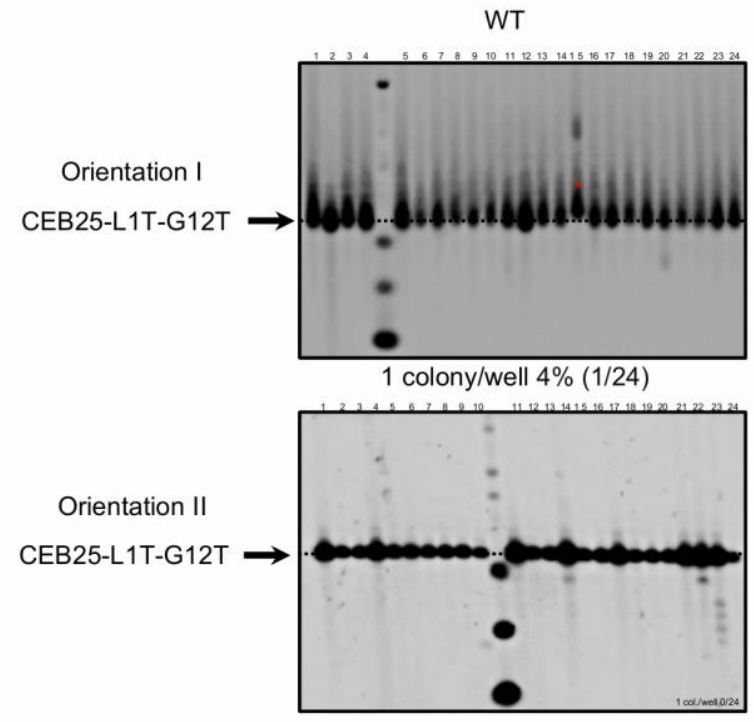

1 colony/well $11 \%(4 / 36)$

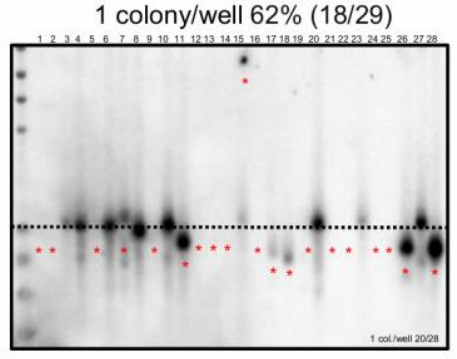

1 colony/well $74 \%(43 / 58)$

rpa1-D223Y

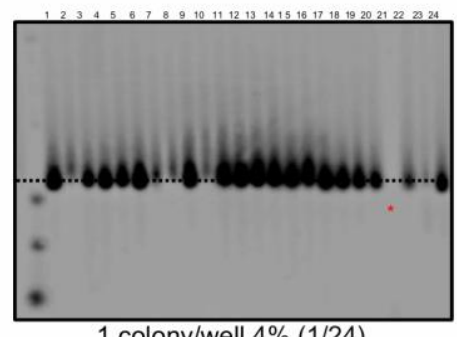

1 colony/well $4 \%(1 / 24)$

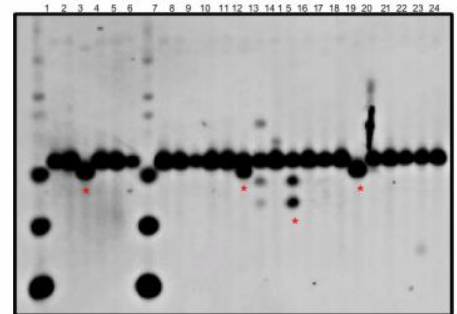

1 colony/well $17 \%(4 / 24)$

FIGURE 7: RPA is required to stabilize CEB25-L1T in fission yeast. (A) Map of the CEB25-L1T insertion within chromosome II at $14 \mathrm{~kb}$ from the ARS-II1964 and at $11 \mathrm{~kb}$ from the ARS-II-1983. (B) Genomic DNA from cells containing CEB25-L1T in orientation 1 and 2 was digested by Pvull and southern blotted. Membranes were hybridized with CEB25 probe. (C) Genomic DNA from cells containing mutated CEB25-L1T-G12T in orientation 1 and 2 was digested by Pvull and southern blotted. Membranes were hybridized with a CEB25-L1T probe. The number of colonies analysed per well, the percentage of rearrangement frequencies, and the total number of colonies are indicated. Red stars mark unstable events. We calculated the frequency of instability by monitoring the size and the intensity of the CEB25. We considered that CEB25 was unstable when the intensity of the short or high band was superior to the one of the parental band or when the band disappears. The number of colonies analysed per well, the percentage of rearrangement frequencies, and the total numbers of colonies are indicated in Table 1. 
A

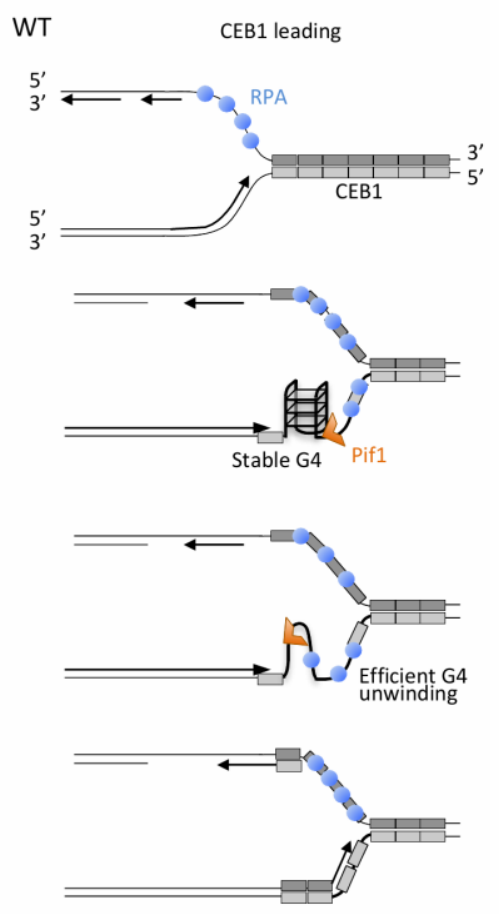

CEB1 size conservation
CEB1 lagging
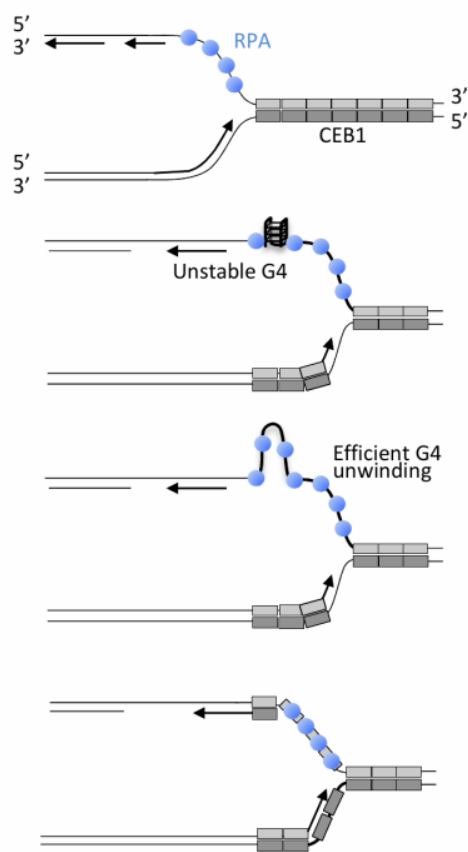

CEB1 size conservation
B

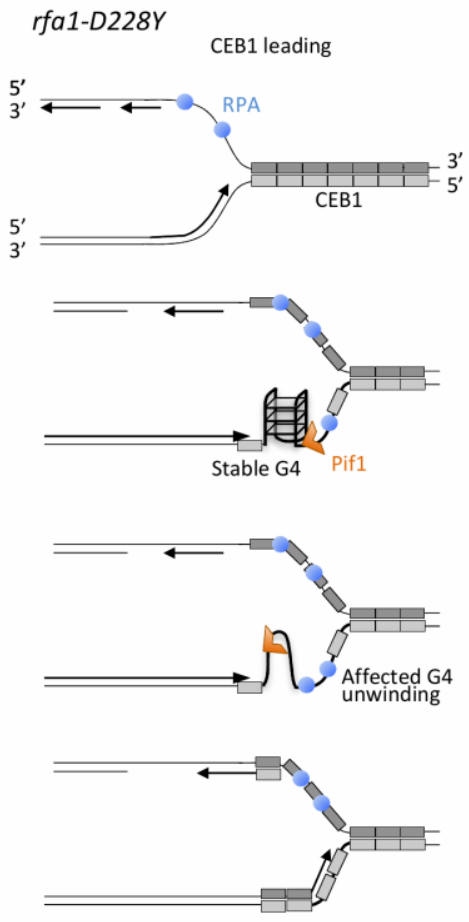

CEB1 rearrangement(s)

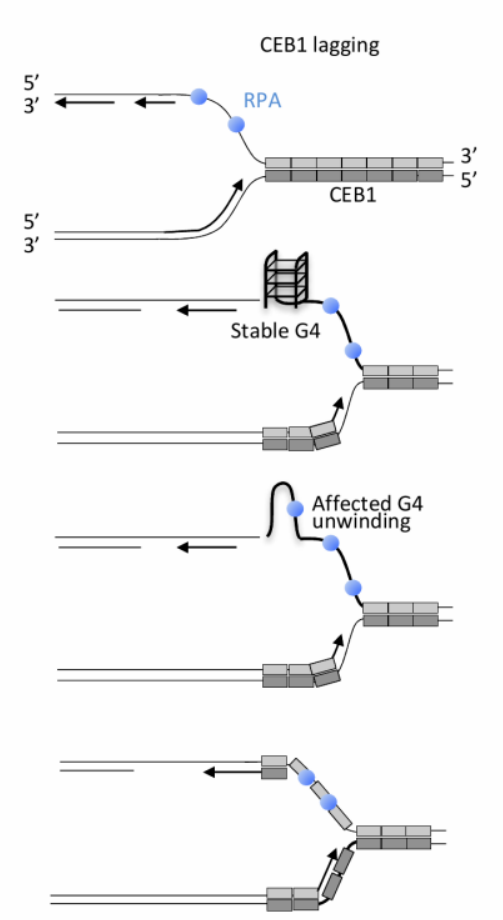

CEB1 rearrangement(s)
CEB1 lagging (PIF1 overexpression)

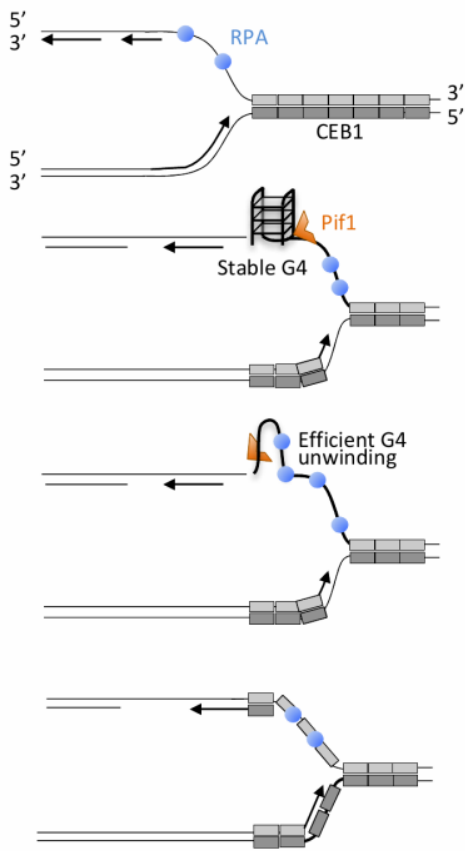

CEB1 size conservation

FIGURE 8: Mechanistic model for the unfolding of CEB1-G-rich structures by RPA and Pif1. (A) In WT cells, when the G-rich strand (light grey) is on leading strand (left part, CEB1 leading), stable G-quadruplexes structures are efficiently removed by Pif1, as proposed by Lopes and colleagues [33]. At the leading strand, RPA may cooperate with Pif1 either by preventing the refolding of the G4 structure or by recruiting Pif1 to the leading strand. When the G-rich strand (light grey) is replicated by the lagging polymerase (right part, CEB1 lagging) binding of RPA to the G-rich strand prevents formation of stable G-rich secondary structures. In this context, Pif1 is dispensable. (B) In the rfa1-D228Y mutant, at the leading strand (left part), the reduced affinity of RPA for G-rich ssDNA either reduce the ability of RPA to prevent the refolding of the G4 structure or decrease Pif1 recruitment. The decrease in RPA affects G4-unwinding and leads to the formation of CEB1 rearrangements. At the lagging strand (middle part), the decrease affinity of RPA for Grich ssDNA facilitates the formation of stable G-rich structures, affects their unwinding, and generates CEB1 instability. In such a case, overexpression of Pif1 (right part) can efficiently unwind G-rich structures, leading to CEB1 size conservation. 
cessed by Pif1 indicating that RPA and Pif1 have redundant G-quadruplex-processing activities at the lagging strand and cooperate (Figure 8).

Pif1 binds to G4 motifs with no preference for leading and lagging strand templates [57] but Pif1 is essential only for efficient replication through lagging strand G4s [60]. Consequently, the distinct behaviour of Pif1 at the leading and the lagging strands may be due to different conformations of the respective G-quadruplexes, affecting their folding and/or their processing due to the occupancy rate of RPA. Another possibility could be that the blocking G-quadruplex structures are better tolerated and bypassed by the lagging strand replication machinery due to its ability to prime DNA synthesis downstream of G-quadruplexes. However, this seems improbable because CEB1 instability in rfa1-D228Y cells is fully suppressed by Pif1 overexpression suggesting that the blocking structures are present and that the helicase activity of Pif1 is still required to remove G4-structures and rescue CEB1 stability.

How exactly Pif1 is recruited at the G-rich motifs and at G-quadruplexes that form at CEB1 during replication? One possibility is that Pif1 travels with the replication fork and facilitates replication by processing G-quadruplex structures at the leading-CEB1. Indeed, Pif1 interacts with PCNA and Cac1, the large CAF-1 subunit, which preferentially assembles nucleosome onto replicating DNA $[60,61]$. Thus, Pif1 may be targeted to the replication fork by its ability to interact with PCNA or the histone chaperone Cac1 and preserves genome stability by acting at G-rich motifs at the leading-CEB1. Alternatively, Pif1 could be directly recruited to G-rich motifs and G-quadruplex structures. Mms1 is a G4-DNA-binding protein that helps replication fork progression at G4 and Pif1 binding to specific G4 structures $[22,37]$. We show that Mms1 is not required to maintain CEB1 stability at both leading- and lagging-CEB1 revealing that $\mathrm{G} 4$ structures targeted by $\mathrm{Mms1}$ are not deleterious for CEB1 stability. Our results are in good agreement with previous observations showing that Mms1 supports Pif1 function at G4 motifs only on the lagging strand [22, 37], whereas Pif1 is not required for CEB1 stabilisation [33]. Here we show that RPA recruits Pif1 to CEB1. We report that Pif1 associates with RPA and that this association is affected in the rfa1-D228Y mutant and by DNA digestion, suggesting that Pif1-RPA association relies on specific DNA structures. In addition we show that rtt105A mutation, which decreases the level of RPA associated with SSDNA (as the rfa1-D228Y mutation) [44, 45], strongly affects CEB1 stability at both leading and lagging strands. These results reveal the importance of Rtt105 in promoting the replication of G4-forming CEB1 minisatellite, during leading strand and lagging strand synthesis, and confirm that the level of RPA is crucial for CEB1 stability. RPA protects and stabilizes ssDNA susceptible to secondary structure formation. It is possible that the ability of RPA to directly bind to Pif1 could be important to recruit Pif1 at specific G-rich sequences/structures and/or to stimulate Pif1 activity at G-rich motifs and G-quadruplex structures.

Importantly, the results obtained in $S$. cerevisiae with the rfa1-D228Y mutant were confirmed in S. pombe with the rpa1-D223Y mutant. The effects of the rpa1-D223Y mutant were even more pronounced in $S$. pombe. This may be due to the fact that fission yeast lacks a functional ortholog of the Pif1 helicase. Indeed Pfh1 behaves more closely to the budding yeast Rrm3 helicase than to Pif1 [56]. This may account for the even more prominent role of RPA complex in $\mathrm{G} 4$ unwinding in fission yeast. This assumption is also supported by the fact that the rpa1-D223Y mutation has a stronger effect at telomeres in $S$. pombe than its counterpart in S. cerevisiae [38,62]. Interestingly, we observed expansions of the CEB25-L1T even in the WT strain, especially when CEB25-L1T was preferentially replicated by the lagging machinery. This result opens new avenues to use fission yeast as a model organism to study expansions of G-rich sequences that have been associated with neurological diseases.

RPA interacts with RNase $\mathrm{H} 1$ and colocalizes with it at R-loops raising the possibility that RPA could recruit and/or stimulate RNase $\mathrm{H} 1[41,53]$. We observed that the interaction between RPA and RNase $\mathrm{H} 1$ is stimulated by the presence of DNA and is slightly affected in rfa1-D228Y cells. Furthermore, Pif1 regulates R-loop formation and potentially complements RNase $\mathrm{H}$ for R-loop resolution [51, 52]. We found that RNase $\mathrm{H} 1$ overexpression did not rescue CEB1 stability in pif1 $1, r f a 1-D 228 Y$, and $r t 1105 \Delta$ mutants. These results suggest that R-loops are likely not responsible of CEB1 instability in these mutants, indicating that CEB1 instability is not due to transcription. Collectively, our results add new insights about the role of RPA as a general sensor of secondary structures and regulator of genomic stability. RPA acts through direct interactions with proteins acting at the level of specific secondary structures, which are hotspots of genomic instability. This mode of RPA action is conserved between yeasts and humans.

\section{MATERIALS AND METHODS}

\section{Strains and growth conditions}

Strains used in this study are listed in Table 2. Yeast strains containing CEB1-1.8 I (orientation I), CEB1-1.8 II (orientation II), or CEB1Gmut-1.7 were mated with pif1 $\Delta$, rfa1-D228Y, pif1 $\triangle$

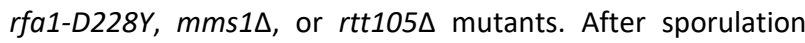
and identification of the four resulting spores, the strains of interest were plated on rich medium at $30^{\circ} \mathrm{C}$ to obtain isolated colonies. Individual clones were grown in rich liquid culture at $30^{\circ} \mathrm{C}$ until stationary phase. For PIF1 overexpression experiments, WT cells and rfa1-D228Y cells were transformed with the centromeric plasmids pVS45 (expressing the nuclear form of Pif1 under the control of the GAL1 promoter) and pSH380 (a pRS315-derived vector control) provided by Virginia Zakian [43], then mated with leading-CEB1 (orientation I), or laggingCEB1 (orientation II) cells. Individual clones were grown in SDLeu ( $2 \%$ glucose), or SGal-Leu ( $2 \%$ galactose) media for repression or overexpression of PIF1, when mentioned.

The $0.62 \mathrm{~kb}$ CEB25-L1T and CEB25-L1T-G12T were cloned into pJK148 integrative plasmid in both orientations at Not1 site, from plasmid pPA84 and pPA84-G12T (A. Nicolas's Laboratory), respectively. The corresponding plasmids were linearized by Nde1 and transformed into S. pombe cells. To check the insertion of CEB25 at the leu1 locus, genomic DNA was digested by $\mathrm{Xbal}$ a nd southern blotted using a leu1 probe. 
Table2. Strains used in this study.

\begin{tabular}{|c|c|c|}
\hline S. cerevisiae strains & Genotype & Origin \\
\hline ORT6119-4 & MATa CEB1-1.8 I-ARS305 & Nicolas A. \\
\hline ORT6135-36 & MATa CEB1-1.8 II-ARS305 & Nicolas A. \\
\hline ORT6157-1 & MATa CEB1-1.7 Gmut-ARS305 & Nicolas A. \\
\hline LM361 & diploïd CEB1-1.8 I rfa1-D228Y/RFA1 pif1::Kan/PIF1 & This study \\
\hline LM411 & diploïd CEB1-1.8 // rfa1-D228Y/RFA1 pif1::Kan/PIF1 & This study \\
\hline LM349 & diploïd CEB1-1.7 Gmut rfa1-D228Y/RFA1 pif1::Kan/PIF1 & This study \\
\hline LM396 & diploïd CEB1-1.8 I rfa1-D228Y/RFA1 + pVS45 (GAL::PIF1) & This study \\
\hline LM398 & diploïd CEB1-1.8 // rfa1-D228Y/RFA1 + pVS45 (GAL::PIF1) & This study \\
\hline LM401 & diploïd CEB1-1.8 I rfa1-D228Y/RFA1 + pSH380 (empty vector) & This study \\
\hline LM404 & diploïd CEB1-1.8 // rfa1-D228Y/RFA1 + pSH380 (empty vector) & This study \\
\hline YVC600 & diploïd CEB1-1.8 I mms1::TRP1/MMS1 & This study \\
\hline YVC601 & diploïd CEB1-1.8 // mms1::TRP1/MMS1 & This study \\
\hline YVC602 & diploïd CEB1-1.8 I rtt105::Kan/RTT105 & This study \\
\hline YVC603 & diploïd CEB1-1.8 /I rtt105::Kan /RTT105 & This study \\
\hline YVC604 & diploïd CEB1-1.8 I rtt105::Kan/RTT105 GAL::RNH1::natMX/RNH1 & This study \\
\hline YVC605 & diploïd CEB1-1.8 I/ rtt105::Kan/RTT105 GAL::RNH1::natMX /RNH1 & This study \\
\hline YVC606 & diploïd CEB1-1.8 I pif1::Kan/PIF1 GAL::RNH1::natMX /RNH1 & This study \\
\hline YVC607 & diploïd CEB1-1.8 I rfa1-D228Y/RFA1 GAL::RNH1::natMX /RNH1 & This study \\
\hline YVC608 & diploïd CEB1-1.8 // rfa1-D228Y/RFA1 GAL::RNH1::natMX/RNH1 & This study \\
\hline LM140 & MATa rfa1-D228Y Pif1-myc::KanMX6 & This study \\
\hline W1042-7C & MATa can1-100,x SUP4-o::HIS3::pWJ317-CAN1-URA3 rfa1-D228Y & Rothstein R \\
\hline LM301 & pif1::KanMX6 rfa1-D228Y & This study \\
\hline LM340 & pif1::KanMX6 & This study \\
\hline YBL103 & his $3 \Delta 1$; leu2 $\Delta 0$; ura3 $\Delta 0$; met15 $\Delta 0$; pGal-3HA-RNH1::NAT & Luke B. \\
\hline LM407 & diploïd pGal-3HA-RNH1::natMX rfa1-D228Y/RFA1 & This study \\
\hline S. pombe strains & Genotype & Origin \\
\hline JA961 & h- leu1-32 ura4-D18 leu1+::pJK148-CEB25 orientation 1 & This study \\
\hline JA963 & h- leu1-32 ura4-D18 leu1+::pJK148-CEB25-G12T orientation 1 & This study \\
\hline JA947 & h- leu1-32 ura4-D18 leu1+::pJK148-CEB25 orientation 2 & This study \\
\hline JA948 & h- leu1-32 ura4-D18 leu1+::pJK148-CEB25-G12T orientation 2 & This study \\
\hline SC387 & $h+$ leu1-32 ura4-D18 ade6-M210 rad11-D223Y & Ueno M. \\
\hline
\end{tabular}

All LM strains used are derivatives of W303-1B. All YVC strains used are derivatives of W303.

Spore colonies were generated from the diploid strains.

Correct and unique insertion of CEB25 at this locus generated two fragments of 3 and $14.4 \mathrm{~kb}$. To check the correct size of CEB25-L1T and CEB25-L1T-G12T, a second digestion by Pvull on genomic DNA was performed on selected clones. Southern blot hybridized by a CEB25-L1T probe (Not1-fragment from pPA84 plasmid), revealed a fragment of $1.1 \mathrm{~kb}$. Fission yeast strains containing CEB25-L1T and CEB25-L1T-G12T were then mated with the rpa1-D223Y mutant. After sporulation and identification of the resulting spores, the stability of the CEB25 was controlled a second time by Southern blot after digestion of genomic DNA by Pvull. The strains of interest were plated on rich medium to obtain isolated colonies and individual clones were further grown in rich liquid culture at $32^{\circ} \mathrm{C}$ until stationary phase.

\section{Southern blot analyses}

Genomic DNA was prepared from $1 \times 10^{8}$ cells according to standard protocols and digested with Apal/Xhol, Apal/Ncol and Apal/Sacll (New England Biolabs) for analysis of CEB1-1.8 I, CEB1-1.8 II and CEB1Gmut-1.7, respectively $[31,63]$. The di- gested DNA was resolved in $1 \%$ agarose gel and blotted onto Hybond-XL membrane (GE Healthcare). After transfer, the membrane was cross-linked with UV and hybridized with CEB1-0.6 and CEB1 Gmut probes for CEB1-1.8 and CEB1Gmut 1.7 , respectively. ${ }^{32} \mathrm{P}$ labelling of DNA probes was performed by random priming using Klenow fragment exonuclease (New England Biolabs), in presence of $\left[\alpha^{-32} \mathrm{P}\right]-\mathrm{CTP}$ and hybridizations were performed in Church buffer at $55^{\circ} \mathrm{C}$. Radioactive signals were detected using a BIORAD molecular imager FX.

\section{Co-Immunoprecipitation experiment}

Yeast cells were grown at $30^{\circ} \mathrm{C}$ in YPD to $\mathrm{OD}_{600}=0.8$. Extracts were lysed with glass-beads in TMG-50 $(10 \mathrm{mM}$ Tris $\mathrm{HCl}$ pH8.0, $1 \mathrm{mM} \mathrm{MnCl}, 10 \%$ (v/v) glycerol, $50 \mathrm{mM} \mathrm{NaCl}, 0.1 \mathrm{mM} \mathrm{DTT}$ ) containing a protease inhibitor cocktail (Calbiochem), MG132 (Sigma Aldrich), and 0.5\% (v/v) Tween-20. Pif1-Myc and Rnh1HA proteins were immunoprecipitated with $9 \mathrm{E} 10$ monoclonal anti-Myc antibody (Santa Cruz, CA) and 12CA5 anti-HA monoclonal antibody (Roche), respectively. The presence of Rfa1 
and Rfa2 in the IP was checked with anti-Rfa1 and anti-Rfa2 antibodies (Agrisera, Sweden).

\section{RNA isolation and analysis}

Total RNA was isolated from $2 \times 10^{8}$ cells using the hot phenol method [64]. Total RNA was treated with RNase-free DNase I (Qiagen) and reverse transcribed using the Superscriptlll reverse transcriptase (Life technologies) with random hexanucleotide primers (Sigma-aldrich). Quantitative PCR amplification of CDNA was carried out using the SYBR Premix Ex Taq II (Ozyme) with these cycling parameters: 1 cycle at $95^{\circ} \mathrm{C}$ for 30 $\mathrm{sec}$, followed by $45 \mathrm{cycles}$ at $95^{\circ} \mathrm{C}$ for $10 \mathrm{sec}, 58^{\circ} \mathrm{C}$ for $15 \mathrm{sec}$, and $72^{\circ} \mathrm{C}$ for $20 \mathrm{sec}$, and a final extension step at $72^{\circ} \mathrm{C}$ for 5 min. PIF1 cDNA was quantified by using the oligonucleotides Pif1-forward (5'-CTGAAAACTCATTTGACCAG-3') and Pif1reverse (5'-GCAATCTTTTCTCCAAATTGC-3'). ACT1 cDNA was quantified by using the oligonucleotides Act1-forward (5'CTATGTTACGTCGCCTTGGA-3') and Act1-reverse (5'TTTGGTCAATACCGGCAGAT-3').

\section{ACKNOWLEDGMENTS}

We are very grateful to Alain Nicolas (Institut Curie) and Judith Lopes (Museum d'Histoire Naturelle) for the gift of

\section{REFERENCES}

1. Brill SJ and Stillman B (1991). Replication factor-A from Saccharomyces cerevisiae is encoded by three essential genes coordinately expressed at $S$ phase. Genes Dev 5(9): 1589-1600. doi: 10.1101/gad.5.9.1589

2. Chen R and Wold MS (2014). Replication protein A: single-stranded DNA's first responder: dynamic DNA-interactions allow replication protein $A$ to direct single-strand DNA intermediates into different pathways for synthesis or repair. Bioessays 36(12): 1156-1161. doi: 10.1002/bies.201400107

3. Iftode C, Daniely $Y$ and Borowiec JA (1999). Replication protein A (RPA): the eukaryotic SSB. Crit Rev Biochem Mol Biol 34(3): 141-180. doi: 10.1080/10409239991209255

4. Chen $\mathrm{H}$, lisby $\mathrm{M}$ and Symington LS (2013). RPA Coordinates DNA End Resection and Prevents Formation of DNA Hairpins. Mol Cell 50(4): 589-600. doi: 10.1016/j.molcel.2013.04.032

5. Bae SH, Bae KH, Kim JA and Seo YS (2001). RPA governs endonuclease switching during processing of Okazaki fragments in eukaryotes. Nature 412(6845): 456-461. doi: 10.1038/35086609

6. Burgers PMJ (2009). Polymerase dynamics at the eukaryotic DNA replication fork. J Biol Chem 284(7): 4041-4045. doi: 10.1074/jbc.R800062200

7. Kao H-I, Veeraraghavan J, Polaczek P, Campbell JL and Bambara RA (2004). On the roles of Saccharomyces cerevisiae Dna2p and Flap endonuclease 1 in Okazaki fragment processing. J Biol Chem 279(15): 15014-15024. doi: 10.1074/jbc.M313216200

8. Stith CM, Sterling J, Resnick MA, Gordenin DA and Burgers PM (2008). Flexibility of eukaryotic Okazaki fragment maturation through regulated strand displacement synthesis. J Biol Chem 283(49): 3412934140. doi: 10.1074/jbc.M806668200

9. Rossi ML, Pike JE, Wang W, Burgers PM, Capbell JL and Bambara RA (2008). Pif1 helicase directs eukaryotic Okazaki fragments toward the two-nuclease cleavage pathway for primer removal. J Biol Chem 283(41): 27483-27493. doi: 10.1074/jbc.M804550200
CEB1-1.8 I, CEB1-1.8 II, and CEB1Gmut-1.7 strains, and for advices. We thank Brian Luke for the plasmid and the strain overexpressing RNH1. We also thank Nagham Ghaddar. V.G. is supported by the "Ligue Nationale Contre le Cancer (Equipe Labellisée)".

\section{CONFLICT OF INTEREST}

The authors declare that they have no conflict of interest.

\section{COPYRIGHT}

(C) 2020 Maestroni et al. This is an open-access article released under the terms of the Creative Commons Attribution (CC BY) license, which allows the unrestricted use, distribution, and reproduction in any medium, provided the original author and source are acknowledged.

Please cite this article as: Laetitia Maestroni, Julien Audry, Pierre Luciano, Stéphane Coulon, Vincent Géli and Yves Corda (2020) RPA and Pif1 cooperate to remove G-rich structures at both leading and lagging strand. Cell Stress 4(3): 48-63. doi: $10.15698 /$ cst2020.03.214

10. Pike JE, Burgers PMJ, Campbell JL and Bambara RA (2009). Pif1 helicase lengthens some Okazaki fragment flaps necessitating Dna2 nuclease/helicase action in the two-nuclease processing pathway. J Biol Chem 284(37): 25170-25180. doi: 10.1074/jbc.M109.023325

11. Levikova $M$ and Cejka $P$ (2015). The Saccharomyces cerevisiae Dna2 can function as a sole nuclease in the processing of Okazaki fragments in DNA replication. Nucleic Acids Res 43(16): 7888-7897. doi: $10.1093 /$ nar/gkv710

12. Ayyagari R, Gomes XV, Gordenin DA and Burgers PMJ (2003). Okazaki fragment maturation in yeast. I. Distribution of functions between FEN1 AND DNA2. J Biol Chem 278(3): 1618-1625. doi: 10.1074/jbc.M209801200

13. Gloor JW, Balakrishnan L, Campbell JL and Bambara RA (2012). Biochemical analyses indicate that binding and cleavage specificities define the ordered processing of human Okazaki fragments by Dna2 and FEN1. Nucleic Acids Res 40(14): 6774-6786. doi: $10.1093 / \mathrm{nar} / \mathrm{gks} 388$

14. Zhou C, Pourmal S and Pavletich NP (2015). Dna2 nucleasehelicase structure, mechanism and regulation by Rpa. Elife 4: e09832. doi: 10.7554/eLife.09832

15. Safa L, Gueddouda NM, Thiébaut F, Delagoutte E, Petruseva I, Lavrik O, Mendoza O, Bourdoncle A, Alberti P, Riou J-F and Saintomé C (2016). 5' to 3' Unfolding Directionality of DNA Secondary Structures by Replication Protein A: G-quadruplexes and duplexes. J Biol Chem 291(40): 21246-21256. doi: 10.1074/jbc.M115.709667

16. Duquette ML, Handa P, Vincent JA, Taylor AF and Maizels N (2004). Intracellular transcription of G-rich DNAs induces formation of G-loops, novel structures containing G4 DNA. Genes Dev 18(13): 1618-1629. doi: $10.1101 /$ gad. 1200804

17. Bochman ML, Paeschke $K$ and Zakian VA (2012). DNA secondary structures: stability and function of G-quadruplex structures. Nat Rev Genet 13(11): 770-780. doi: 10.1038/nrg3296

18. Rhodes D and Lipps HJ (2015). G-quadruplexes and their regulatory roles in biology. Nucleic Acids Res 43(18): 8627-8637. doi: 10.1093/nar/gkv862 
19. Mendoza O, Bourdoncle A, Boulé J-B, Brosh RM and Mergny J-L (2016). G-quadruplexes and helicases. Nucleic Acids Res 44(5): 19892006. doi: 10.1093/nar/gkw079

20. Gellert M, Lipsett MN and Davies DR (1962). Helix formation by guanylic acid. Proc Natl Acad Sci USA 48: 2013-2018. doi: 10.1073/pnas.48.12.2013

21. Williamson JR, Raghuraman MK and Cech TR (1989). Monovalent cation-induced structure of telomeric DNA: the G-quartet model. Cell 59(5): 871-880. doi: 10.1016/0092-8674(89)90610-7

22. Wanzek K, Schwindt E, Capra JA and Paeschke K (2017). Mms1 binds to G-rich regions in Saccharomyces cerevisiae and influences replication and genome stability. Nucleic Acids Res 45(13): 7796-7806 doi: 10.1093/nar/gkx467

23. Götz S, Pandey S, Bartsch S, Juranek S and Paeschke K (2019). A Novel G-Quadruplex Binding Protein in Yeast-Slx9. Molecules 24(9): 1774. doi: 10.3390/molecules24091774

24. Sauer $M$ and Paeschke $K$ (2017). G-quadruplex unwinding helicases and their function in vivo. Biochem Soc Trans 45(5): 1173-1182. doi: 10.1042/BST20170097

25. Lerner LK and Sale JE (2019). Replication of G Quadruplex DNA. Genes 10(2): 95. doi: 10.3390/genes10020095

26. Paeschke K, Bochman ML, Garcia PD, Cejka P, Friedman KL, Kowalczykowski SC and Zakian VA (2013). Pif1 family helicases suppress genome instability at G-quadruplex motifs. Nature 497(7450): 458462. doi: $10.1038 /$ nature 12149

27. Brázda V, Hároníková L, Liao JCC and Fojta M (2014). DNA and RNA quadruplex-binding proteins. Int J Mol Sci 15(10): 17493-17517. doi: 10.3390/ijms151017493

28. Castillo Bosch P, Segura-Bayona S, Koole W, van Heteren JT, Dewar $\mathrm{JM}$, Tijsterman $\mathrm{M}$ and Knipscheer $\mathrm{P}$ (2014). FANCJ promotes DNA synthesis through G-quadruplex structures. Embo J 33(21): 2521-2533. doi: 10.15252/embj.201488663

29. Piazza A, Cui X, Adrian M, Samazan F, Heddi B, Phan AT and Nicolas AG (2017). Non-Canonical G-quadruplexes cause the hCEB1 minisatellite instability in Saccharomyces cerevisiae. Elife 6: e26884. doi: 10.7554/eLife. 26884

30. Wu CG and Spies M (2016). G-quadruplex recognition and remodeling by the FANCJ helicase. Nucleic Acids Res 44(18): 8742-8753. doi: 10.1093/nar/gkw574

31. Ribeyre C, lopes J, Boulé JB, Piazza A, Guédin A, Zakian VA, Mergny $\mathrm{JL}$ and Nicolas A (2009). The yeast Pif1 helicase prevents genomic instability caused by G-quadruplex-forming CEB1 sequences in vivo. Plos Genet 5(5): e1000475. doi: 10.1371/journal.pgen.1000475

32. Piazza A, Boulé JB, Lopes J, Mingo K, Largy E, Teulade-Fichou MP and Nicolas $A$ (2010). Genetic instability triggered by G-quadruplex interacting Phen-DC compounds in Saccharomyces cerevisiae. Nucleic Acids Res 38(13): 4337-4348. doi: 10.1093/nar/gkq136

33. Lopes J, Piazza A, Bermejo R, Kriegsman B, Colosio A, TeuladeFichou M-P, Foiani $M$ and Nicolas A (2011). G-quadruplex-induced instability during leading-strand replication. Embo J 30(19): 40334046. doi: 10.1038/emboj.2011.316

34. Piazza A, Serero A, Boulé J-B, Legoix-Né P, Lopes J and Nicolas A (2012). Stimulation of gross chromosomal rearrangements by the human CEB1 and CEB25 minisatellites in Saccharomyces cerevisiae depends on G-quadruplexes or Cdc13. PLoS Genet 8(11): e1003033. doi: 10.1371/journal.pgen.1003033

35. Yu C, Gan H, Han J, Zhou Z-X, Jia S, Chabes A, Farrugia G, Ordog T and Zhang $Z$ (2014). Strand-Specific Analysis Shows Protein Binding at Replication Forks and PCNA Unloadingfrom Lagging Strands when Forks Stall. Mol Cell 56(4): 1-13. doi: 10.1016/j.molcel.2014.09.017
36. Rossi SE, Foiani M and Giannattasio M (2018). Dna2 processes behind the fork long ssDNA flaps generated by Pif1 and replicationdependent strand displacement. Nat Commun 9: 4830-11. doi: 10.1038/s41467-018-07378-5

37. Schwindt $E$ and Paeschke $K$ (2018). Mms1 is an assistant for regulating G-quadruplex DNA structures. Curr Genet 64(3): 535-540. doi: 10.1007/s00294-017-0773-9

38. Audry J, Maestroni L, Delagoutte E, Gauthier T, Nakamura TM, Gachet $Y$, Saintomé C, Géli V and Coulon S (2015). RPA prevents G-rich structure formation at lagging-strand telomeres to allow maintenance of chromosome ends. Embo J 34(14): 1942-1958. doi: 10.15252/embj. 201490773

39. Smith J and Rothstein R (1995). A mutation in the gene encoding the Saccharomyces cerevisiae single-stranded DNA-binding protein Rfa1 stimulates a RAD52-independent pathway for direct-repeat recombination. Mol Cell Biol 15(3): 1632-1641. doi: 10.1128/mcb.15.3.1632

40. Deng SK, Gibb B, de Almeida MJ, Greene EC and Symington LS (2014). RPA antagonizes microhomology-mediated repair of DNA double-strand breaks. Nat Struct Mol Biol 21(4): 405-412. doi: 10.1038/nsmb. 2786

41. Nguyen HD, Yadav T, Giri S, Saez B, Graubert TA and Zou L (2017). Functions of Replication Protein A as a Sensor of R Loops and a Regulator of RNaseH1. Mol Cell 65(5): 832-847.e4. doi 10.1016/j.molcel.2017.01.029

42. Chang $M$, Luke B, Kraft C, Li Z, Peter M, Lingner J and Rothstein R (2009). Telomerase Is Essential to Alleviate Pif1-Induced Replication Stress at Telomeres. Genetics 183(3): 779-791. doi: 10.1534/genetics.109.107631

43. Vega LR, Phillips JA, Thornton BR, Benanti JA, Onigbanjo MT, Toczyski DP and Zakian VA (2007). Sensitivity of yeast strains with long G-tails to levels of telomere-bound telomerase. Plos Genet 3(6): e105. doi: 10.1371/journal.pgen.0030105

44. Li S, Xu Z, Xu J, Zuo L, Yu C, Zheng P, Gan H, Wang X, Li L, Sharma S, Chabes A, Li D, Wang S, Zheng S, Li J, Chen X, Sun Y, Xu D, Han J, Chan K, Qi Z, Feng J and Li Q (2018). Rtt105 functions as a chaperone for replication protein A to preserve genome stability. Embo J 37(17): e99154. doi: 10.15252/embj.201899154

45. Li S, Dong Z, Yang S, Feng J and Li Q (2019). Chaperoning RPA during DNA metabolism. Curr Genet 65(4): 857-864. doi: 10.1007/s00294-019-00945-3

46. Thomas M, White RL and Davis RW (1976). Hybridization of RNA to double-stranded DNA: formation of R-loops. Proc Natl Acad Sci USA 73: 2294-2298. doi: 10.1073/pnas.73.7.2294

47. Daniels GA and Lieber MR (1995). RNA:DNA complex formation upon transcription of immunoglobulin switch regions: implications for the mechanism and regulation of class switch recombination. Nucleic Acids Res 23(24): 5006-5011. doi: 10.1093/nar/23.24.5006

48. Šviković S, Crisp A, Tan-Wong SM, Guilliam TA, Doherty AJ, Proudfoot NJ, Guilbaud G and Sale JE (2019). R-loop formation during $S$ phase is restricted by PrimPol-mediated repriming. Embo $\mathbf{J} 38(3)$ : 5286. doi: 10.15252/embj.201899793

49. Kim N and Jinks-Robertson S (2011). Guanine repeat-containing sequences confer transcription-dependent instability in an orientation-specific manner in yeast. DNA Repair 10(9): 953-960. doi: 10.1016/j.dnarep.2011.07.002

50. Zhou R, Zhang J, Bochman ML, Zakian VA and Ha T (2014). Periodic DNA patrolling underlies diverse functions of Pif1 on R-loops and Grich DNA. Elife 3: e02190. doi: 10.7554/eLife.02190 
51. Tran PLT, Pohl TJ, Chen CF, Chan A, Pott S and Zakian VA (2017). PIF1 family DNA helicases suppress R-loop mediated genome instability at tRNA genes. Nat Commun 8: 15025-10. doi: 10.1038/ncomms15025

52. Pohl TJ and Zakian VA (2019). Pif1 family DNA helicases: A helpmate to RNase H? DNA Repair 84: 102633. doi: 10.1016/j.dnarep.2019.06.004

53. Gavin A-C, Bösche $M$, Krause $R$, Grandi $P$, Marzioch $M$, Bauer A, Schultz J, Rick JM, Michon A-M, Cruciat C-M, Remor M, Höfert C, Schelder $M$, Brajenovic $M$, Ruffner $H$, Merino A, Klein $K$, Hudak $M$, Dickson D, Rudi T, Gnau V, Bauch A, Bastuck S, Huhse B, Leutwein C, Heurtier M-A, Copley RR, Edelmann A, Querfurth E, Rybin V, Drewes G, Raida M, Bouwmeester T, Bork P, Séraphin B, Kuster B, Neubauer $G$ and Superti-Furga $G$ (2002). Functional organization of the yeast proteome by systematic analysis of protein complexes. Nature 415(6868): 141-147. doi: 10.1038/415141a

54. Piazza A, Adrian M, Samazan F, Heddi B, Hamon F, Serero A, Lopes J, Teulade-Fichou M-P, Phan AT and Nicolas A (2015). Short loop length and high thermal stability determine genomic instability induced by G-quadruplex-forming minisatellites. Embo J 34(12): 17181734. doi: 10.15252 /embj. 201490702

55. Wu P-YJ and Nurse P (2009). Establishing the program of origin firing during $S$ phase in fission Yeast. Cell 136(5): 852-864. doi: 10.1016/j.cell.2009.01.017

56. Sabouri N, McDonald KR, Webb CJ, Cristea IM and Zakian VA (2012). DNA replication through hard-to-replicate sites, including both highly transcribed RNA Pol II and Pol III genes, requires the S. pombe Pfh1 helicase. Genes Dev 26(6): 581-593. doi: 10.1101/gad.184697.111

57. Paeschke K, Capra JA and Zakian VA (2011). DNA Replication through G-Quadruplex Motifs Is Promoted by the Saccharomyces cerevisiae Pif1 DNA Helicase. Cell 145(5): 678-691. doi: 10.1016/j.cell.2011.04.015

58. Byrd AK and Raney KD (2015). Fine tuning of a DNA fork by the RecQ helicase. Proc Natl Acad Sci USA 112: 15263-15264. doi: 10.1073/pnas.1520119112

59. Gan H, Yu C, Devbhandari S, Sharma S, Han J, Chabes A, Remus D and Zhang $Z$ (2017). Checkpoint Kinase Rad53 Couples Leading- and Lagging-Strand DNA Synthesis under Replication Stress. Mol Cell 68(2): 446-455. doi: 10.1016/j.molcel.2017.09.018

60. Dahan D, Tsirkas I, Dovrat D, Sparks MA, Singh SP, Galletto R and Aharoni A (2018). Pif1 is essential for efficient replisome progression through lagging strand G-quadruplex DNA secondary structures. Nucleic Acids Res 46(22): 11847-11857. doi: 10.1093/nar/gky1065

61. Monson EK, de Bruin D and Zakian VA (1997). The yeast Cac1 protein is required for the stable inheritance of transcriptionally repressed chromatin at telomeres. Proc Natl Acad Sci USA 94: 1308113086. doi: 10.1073/pnas.94.24.13081

62. Luciano P, Coulon S, Faure V, Corda Y, Bos J, Brill SJ, Gilson E, Simon MN and Géli V (2012). RPA Facilitates Telomerase Activity at Chromosome Ends in Budding and Fission Yeasts. Embo J 31(8): 20342046 doi: 10.1038/emboj.2012.40

63. Lopes J, Ribeyre C and Nicolas A (2006). Complex Minisatellite Rearrangements Generated in the Total or Partial Absence of Rad27/hFEN1 Activity Occur in a Single Generation and Are Rad51 and Rad52 Dependent. Mol Cell Biol 26(17): 6675-6689. doi: 10.1128/MCB.00649-06

64. Schmitt ME, Brown TA and Trumpower BL (1990). A rapid and simple method for preparation of RNA from Saccharomyces cerevisiae. Nucleic Acids Res 18(10): 3091-3092. doi: 10.1093/nar/18.10.3091 